\title{
CLASSIFICATION PROBLEMS FOR SYSTEMS OF FORMS AND LINEAR MAPPINGS
}

UDC 512.64

\author{
V. V. SERGEǏCHUK
}

\begin{abstract}
A method is proposed that allows the reduction of many classification problems of linear algebra to the problem of classifying Hermitian forms. Over the complex, real, and rational fields, classifications are obtained for bilinear forms, pairs of quadratic forms, isometric operators, and selfadjoint operators.

Bibliography: 30 titles.
\end{abstract}

Many problems of linear algebra can be formulated as problems of classifying the representations of a quiver. A quiver is, by definition, a directed graph. A representation of the quiver

is given (see [1], and also [2] and [3]) by assigning to each vertex a vector space and to each arrow a linear mapping of the corresponding vector spaces. For example, the quivers correspond respectively to the classification problem for linear operators (whose solution is the Frobenius normal form), for pairs of linear mappings from one space to another (the matrix pencil problem, solved by Kronecker), and for pairs of linear operators (a classical unsolved problem). The notion of quiver has become central in the theory of finite-dimensional algebras over a field: the modules over an algebra are in one-to-one correspondence with the representations of a certain quiver with relations-the Gabriel quiver of the algbra (see [1] and [4]). The theories of quadratic and Hermitian forms are well developed (see [5] and [6]).

We study here systems of sesquilinear forms and linear mappings, regarding them as representations of a partially directed graph (assigning to a vertex a vector space, to an undirected edge a sesquilinear form, and to a directed edge a linear mapping); and we show that the problem of classifying such representations, over a division ring $K$ of

1980 Mathematics Subject Classification (1985 Revision). Primary 15A21, 15A63, 16A64; Secondary $16 \mathrm{A39}$.

This translation incorporates corrections submitted by the author. 
characteristic $\neq 2$, reduces to the problems of classifying:

$1^{\circ}$ Hermitian forms over certain division rings that are extensions of the center of $K$, and

$2^{\circ}$ representations of a certain quiver.

The quiver representations $2^{\circ}$ are known in the case of the problems of classifying:

1) sesquilinear forms (see, for example, [7]-[10]),

2) pairs of Hermitian forms ([6], [11]-[15]), and

3) isometric and selfadjoint operators in a space with nondegenerate Hermitian form ([6], [10], [16]-[19]).

We solve problems 1)-3) over a field $K$ with involution (possibly trivial), and of characteristic $\neq 2$, up to classification of Hermitian forms over fields that are finite extensions of $K$. This yields a classification of bilinear forms and pairs of quadratic forms over the rationals, since over finite extensions of the rationals classifications have been established for quadratic and Hermitian forms (see [5] and [6]).

We study systems of forms and linear mappings by associating with them the selfadjoint representations of a category with involution. For bilinear forms this method was suggested by Gabriel [7]; for systems of forms and linear mappings, by Roiter [20] (see also [21] and [22]). Another approach to classification problems is proposed in [6] and [23], where quadratic and Hermitian forms are studied on objects of an additive category with involution.

The main results of this paper were previously announced in [24]-[25].

The author wishes to thank A. V. Router for his considerable interest and assistance.

\section{$\S 1$. Selfadjoint representations of a linear category with involution}

In this section we prove what might be called a weak Krull-Schmidt theorem for selfadjoint representations of a linear category with involution. Vector spaces are assumed throughout to be right vector spaces.

By a linear category over a field $P$ is meant a category $\mathscr{C}$ in which for every pair of objects $u, v$ the set of morphisms $\operatorname{Hom}(u, v)$ is a vector space over $P$ and multiplication of morphisms is bilinear. The set of objects in $\mathscr{C}$ will be denoted by $\mathscr{C}_{0}$, the set of morphisms by $\mathscr{C}_{1}$. We define the category $R(\mathscr{C})$ of representations of $\mathscr{C}$, over a division ring $K$ with center $P$, as follows. A representation is a functor $A$ from the category $\mathscr{C}$ to the category $\mathscr{V}$ of finite-dimensional vector spaces over $K$, having finite dimension $\operatorname{dim}(A)=\sum_{u \in \mathscr{C}_{0}} \operatorname{dim}\left(A_{u}\right)<\infty$ and preserving linear combinations: $A_{\alpha a+\beta b}=A_{\alpha} a+$ $A_{\beta} b\left(\alpha, \beta \in \mathscr{E}_{1} ; a, b \in P\right)$. A morphism of representations $f: A \rightarrow B$ is a natural transformation of functors, i.e., a set of linear mappings $f_{u}: A_{u} \rightarrow B_{u}\left(u \in \mathscr{C}_{0}\right)$ such that

$$
f_{v} A_{\alpha}=B_{\alpha} f_{u}, \quad \alpha: u \rightarrow v .
$$

Suppose now that $K$ has an involution $a \mapsto \bar{a}(\overline{\bar{a}}=a, \overline{a+b}=\bar{a}+\bar{b}, \overline{a b}=\bar{b} \bar{a}$; if $K$ is a field, the involution can be trivial). Following [20], we define an involution on each of the categories $\mathscr{C}, \mathscr{V}$ and $R(\mathscr{C})$ :

1. To each object $u \in \mathscr{C}_{0}$ we associate an object $u^{*} \in \mathscr{C}_{0}$, and to each morphism $\alpha: u \rightarrow v$ a morphism $\alpha^{*}: v^{*} \rightarrow u^{*}$ so that $u^{* *}=u \neq u^{*}, \alpha^{* *}=\alpha,(\alpha \beta)^{*}=\beta^{*} \alpha^{*}$, $(\alpha a)^{*}=\alpha^{*} \bar{a}\left(u \in \mathscr{C}_{0} ; \alpha, \beta \in \mathscr{C}_{1} ; a \in P\right.$; note that [20] and [22] allow $\left.u^{*}=u\right)$.

2. To each space $V \in \mathscr{V}$ we associate the adjoint space $V^{*} \in \mathscr{V}$ of all semilinear forms $\varphi: V \rightarrow K$,

$$
\varphi(x+y)=\varphi(x)+\varphi(y), \quad \varphi(x a)=\bar{a} \varphi(x)
$$

$(x, y \in V ; a \in K)$, and to each linear mapping $A: U \rightarrow V$ the adjoint linear mapping $A^{*}: V^{*} \rightarrow U^{*}$, where $A^{*} \varphi=\varphi A$. We identify $V$ and $V^{* *}$. 
3. To each representation $A \in R(\mathscr{C})$ we associate the adjoint representation $A^{0} \in$ $R(\mathscr{C})$, where $A_{u}^{0}=A_{u^{*}}^{*}, A_{\alpha}^{0}=A_{\alpha^{*}}^{*}\left(u \in \mathscr{C}_{0}, \alpha \in \mathscr{C}_{1}\right)$; and to each morphism $f: A \rightarrow B$ the adjoint morphism $f^{0}: B^{0} \rightarrow A^{0}$, where $f_{u}^{0}=f_{u}^{*}$. $\left(u \in \mathscr{C}_{0}\right)$. An isomorphism $f: A \rightarrow$ $B$ of selfadjoint representations is called a congruence if $f^{0}=f^{-1}$.

In $\S 2$ we show that the problems of classifying the systems of sesquilinear forms and linear mappings over a division ring $K$ that satisfy certain relations with coefficients in the center of $K$ can be formulated as problems of classifying selfadjoint representations up to congruence. For the present we limit ourselves to examples.

EXAMPLE 1. $\mathscr{C}_{0}=\left\{u, u^{*}\right\}, \mathscr{C}_{1}=1_{u} P \cup 1_{u^{*}} P \cup\left(\alpha P \oplus \alpha^{*} P\right)$, where $\alpha, \alpha^{*}: u \rightarrow u^{*}$. A selfadjoint representation is given by a pair of adjoint linear mappings $A, A^{*}: U \rightarrow U^{*}$, assigned to the morphisms $\alpha$ and $\alpha^{*}$. The representation determines, in a one-to-one manner, a sesquilinear form $A(x, y)=A(y)(x)$ on the space $U$; congruent representations determine equivalent forms.

EXAMPLE 2. $\mathscr{C}_{0}=\left\{u, u^{*}\right\}, \mathscr{C}_{1}=1_{u} P \cup 1_{u^{*}} P \cup \alpha P$, where $\alpha=\varepsilon \alpha^{*}: u \rightarrow u^{*}(0 \neq \varepsilon \in$ $P)$. A selfadjoint representation determines an $\varepsilon$-Hermitian form $A(x, y)=\varepsilon \overline{A(y, x)}$.

We show now how to obtain a classification, up to congruence, of the selfadjoint representations of a category $\mathscr{C}$, starting with the knowledge of a complete system ind $(\mathscr{C})$ of nonisomorphic direct-sum-indecomposable representations. To begin with, let us replace each representation in ind $(\mathscr{C})$ that is isomorphic to a selfadjoint representation by one that is actually selfadjoint, and denote the set of such by $\operatorname{ind}_{0}(\mathscr{C})$. Denote by $\operatorname{ind}_{1}(\mathscr{C})$ the set consisting of all representations in ind $(\mathscr{C})$ that are isomorphic to their adjoints (but not to a selfadjoint), together with one representation from each pair $\{A, B\} \subset \operatorname{ind}(\mathscr{C})$ such that $A$ is not isomorphic to $A^{0}$ but is isomorphic to $B^{0}$.

In addition, we divide the set $\mathscr{C}_{0}$ into two disjoint subsets $S_{0}$ and $S_{0}^{*}$ such that each pair of adjoint objects $u, u^{*}$ has one member in $S_{0}$, the other in $S_{0}^{*}$.

By the orthogonal sum $A \perp B$ of two selfadjoint representations $A$ and $B$ we shall mean the selfadjoint representation obtained from $A \oplus B$ by specifying for each $v \in S_{0}$ the action of $\varphi+\psi \in A_{v}^{*} \oplus B_{v}^{*}=(A \oplus B)_{v^{*}}$ on $a+b \in A_{v} \oplus B_{v}=(A \oplus B)_{v}$ as follows: $(\varphi+\psi)(a+b)=\varphi(a)+\psi(b)$.

For any representation $A$ we define a selfadjoint representation $A^{+}$, obtained from $A \oplus A^{0}$ by specifying in a similar fashion the action of $\left(A \oplus A^{0}\right)_{v^{*}}=A_{v^{*}} \oplus A_{v}^{*}=A_{v}^{*} \oplus A_{v^{*}}^{* *}$ on $\left(A \oplus A^{0}\right)_{v}=A_{v} \oplus A_{v^{*}}^{*}\left(v \in S_{0}\right)$. Taking into account the interchange of summands in the next to last equality, we have

$$
A_{\alpha}^{+}=\left(\begin{array}{cc}
0 & A_{\alpha}^{0} \\
A_{\alpha} & 0
\end{array}\right), \quad A_{\beta}^{+}=\left(\begin{array}{cc}
A_{\beta} & 0 \\
0 & A_{\beta}^{0}
\end{array}\right), \quad A_{\gamma}^{+}=\left(\begin{array}{cc}
0 & A_{\gamma} \\
A_{\gamma}^{0} & 0
\end{array}\right)
$$

for all $\alpha: u \rightarrow v^{*}, \beta: u \rightarrow v, \gamma: u^{*} \rightarrow v\left(u, v \in S_{0}\right)$.

For any selfadjoint representation $A=A^{0}$ and selfadjoint automorphism $f=f^{0}$ of $A$, we define a selfadjoint representation $A^{f}$ and an isomorphism

$$
\tilde{f}: A^{f} \rightarrow A, \quad \tilde{f} \tilde{f}^{0}=f,
$$

by putting $\tilde{f}_{v}=f_{v}, \tilde{f}_{v^{*}}=1\left(v \in S_{0}\right)$ and $A_{v}^{f}=A_{v}\left(v \in \mathscr{C}_{0}\right), A_{\alpha}^{f}=\tilde{f}_{v}^{-1} A_{\alpha} \tilde{f}_{u}(\alpha: u \rightarrow v)$.

Now suppose $K$ has characteristic $\neq 2$. We show in Lemma 1 that the set $R$ of noninvertible elements of the endomorphism ring $\Lambda=\operatorname{End}(B), B \in \operatorname{ind}_{0}(\mathscr{C})$, is the radical of $\Lambda$. Therefore $T(B)=\Lambda / R$ is a division ring with involution $(f+R)^{0}=f^{0}+R$. For each element $0 \neq a=a^{0} \in T(B)$ choose a fixed automorphism $f_{a}=f_{a}^{0} \in a$ (we can take $f_{a}=\frac{1}{2}\left(f+f^{0}\right)$, where $\left.f \in a\right)$, and define $B^{a}=B^{f_{a}}$. The set of representations $B^{a}$ we shall call the orbit of the representation $B$. For any Hermitian form $\varphi(x)=$ $x_{1}^{0} a_{1} x_{1}+\cdots+x_{r}^{0} a_{r} x_{r}\left(0 \neq a_{i}=a_{i}^{0} \in T(B)\right)$, we put

$$
B^{\varphi(x)}=B^{a_{1}} \perp \cdots \perp B^{a_{r}} .
$$


THEOREM 1. Over a division ring $K$ of characteristic $\neq 2$, every selfadjoint representation of a linear category $\mathscr{C}$ with involution is congruent to an orthogonal sum

$$
A_{1}^{+} \perp \cdots \perp A_{m}^{+} \perp B_{1}^{\varphi_{1}(x)} \perp \cdots \perp B_{n}^{\varphi_{n}(x)},
$$

where $A_{i} \in \operatorname{ind}_{1}(\mathscr{C}), B_{j} \in \operatorname{ind}_{0}(\mathscr{C})$, and $B_{j} \neq B_{j^{\prime}}$ for $j \neq j^{\prime}$. The sum is uniquely determined by the original representation up to permutation of summands and replacement of $B_{j}^{\varphi_{j}(x)}$ by $B_{j}^{\psi_{j}(x)}$, where $\varphi_{j}(x)$ and $\psi_{j}(x)$ are equivalent Hermitian forms over the division ring $T\left(B_{j}\right)$.

REMARK. Theorem 1 in fact holds for any ordinary (i.e., nonlinear) category $\mathscr{C}$ with involution, so long as we understand by a representation a functor $A: \mathscr{C} \rightarrow \mathscr{V}$ that has finite $\operatorname{dimension} \operatorname{dim}(A)=\sum \operatorname{dim}\left(A_{u}\right)$. The $\operatorname{ring} K$ can be replaced by any finitedimensional quasi-Frobenius algebra $F$ with involution over a field of characteristic $\neq 2$ (a representation assigns to an object a finitely generated module over $F$ ). A finitedimensional algebra $F$ is quasi-Frobenius if the regular module $F_{F}$ is injective; over such an algebra the finitely generated modules $M$ and $M^{* *}$ can still be identified.

Theorem 1 reduces the classification, up to congruence, of the selfadjoint representations of the category $\mathscr{C}$, assuming known the representations $\operatorname{ind}_{1}(\mathscr{C})$ and the orbits of the representations ind $0(\mathscr{C})$, to the classification of Hermitian forms over the division rings $T(B), B \in \operatorname{ind}_{0}(\mathscr{C})$. If $K$ is finite-dimensional over its center $Z$, then $T=T(B)$ is finite-dimensional over $Z$ under the natural imbedding of $Z$ in the center of $T$, and the involution on $T$ extends the involution on $Z$.

Suppose, for example, that $K$ is a maximal ordered field; i.e., $1<\left(K_{\text {alg }}: K\right)<\infty$, where $K_{\mathrm{alg}}$ is the algebraic closure of $K$. Then its characteristic is $0, K_{\mathrm{alg}}=K(\sqrt{-1})$, and $K$ has only the trivial involution: the stationary subfield relative to involution must coincide with $K$ (see [26], Chap. VI, $\S 2$, nos. 1, 6 and Exercise 22(d)). By the theorem of Frobenius [27], $T$ is equal to $K, K_{\mathrm{alg}}$, or the algebra $H$ of quaternions over $K$. By the law of inertia [27], if $T=K$, or $T=K_{\text {alg }}$ with nontrivial involution, or $T=H$ with the standard involution (taking a quaternion into the conjugate quaternion), then an Hermitian form over $T$ is equivalent to exactly one form of the form $x_{1}^{0} x_{1}+\cdots+x_{l}^{0} x_{l}-$ $x_{l+1}^{0} x_{l+1}-\cdots-x_{r}^{0} x_{r}$. If $T=H$ with nonstandard involution, then every Hermitian form $\varphi(x)=x_{1}^{0} a_{1} x_{1}+\cdots+x_{r}^{0} a_{r} x_{r}\left(a_{i}=a_{i}^{0} \neq 0\right)$ over $T$ is equivalent to the form $x_{1}^{0} x_{1}+\cdots+x_{r}^{0} x_{r}$, since $a_{i}=b_{i}^{2}=b_{i}^{0} b_{i}$, where $b_{i} \in K\left(a_{i}\right)$ for $a_{i} \notin K$ and $b_{i} \in K(d)$ for $a_{i} \in K$. Here $d=d^{0} \notin K$; the existence of $d$ follows from [27] (Chap. VIII, §11, Proposition 2); and $K\left(a_{i}\right)$ and $K(d)$ are algebraically closed fields with trivial involution.

Suppose $K$ is a finite field. Then $T$ is likewise a finite field, over which an Hermitian form reduces uniquely to the form $x_{1}^{0} t x_{1}+x_{2}^{0} x_{2}+\cdots+x_{r}^{0} x_{r}$, where $t$ is equal to 1 for nontrivial involution on $T$, and $t$ is equal to 1 or a fixed nonsquare for trivial involution ([28], Chap. 1, §8).

Thus, applying Theorem 1, we obtain the following assertion, a special case of which is the law of inertia for quadratic and Hermitian forms.

THEOREM 2. Let $K$ be one of the following division rings of characteristic $\neq 2$.

a) An algebraically closed field with trivial involution, or the algebra of quaternions over a maximal ordered field with nonstandard involution.

b) An algebraically closed field with nontrivial involution,-or the algebra of quaternions over a maximal ordered field with standard involution.

c) A maximal ordered field. The theorem is formulated incorrectly

d) A finite field. if $\mathrm{K}$ is the algebra of quaternions; 
Then over $K$ every selfadjoint reprsentation of a linear category $\mathscr{C}$ with involution is congruent to an orthogonal sum, uniquely determined up to permutation of summands, of representations of the following form (where $A \in \operatorname{ind}_{1}(\mathscr{C})$ and $B \in \operatorname{ind}_{0}(\mathscr{C})$ ):

a) $A^{+}, B$.

b) $A^{+}, B, B^{-1}(-1 \in \operatorname{Aut}(B))$.

c) $A^{+}, B^{t}$, where $t=1$ if $T(B)$ is an algebraically closed field with trivial involution or an algebra of quaternions with nonstandard involution, and $t= \pm 1$ otherwise.

d) $A^{+}, B^{t}$, where $t$ is equal to 1 for nontrivial involution on the field $T(B)$, and is equal to 1 or a fixed nonsquare in $T(B)$ for trivial involution, while for each $B$ the orthogonal sum has at most one summand $B^{t}$ with $t \neq 1$.

REMARK 1. A similar assertion can be made for representations over an algebraic number field $K$, since over division rings that are finite central extensions of such a $K$ the classification of Hermitian forms is known (see [6] or [28], Chap. 1, §8).

REMARK 2. It can be shown that over an algebraically closed field of characteristic $\neq$ 2 , or over a maximal ordered field, a system of tensors of valence $\geq 2$ decomposes uniquely, up to isomorphism of summands, into a direct sum of indecomposable subsystems. For a system of valence 2 this follows from Theorem 2 (see $\S 2$ ). Over an algebraically closed field of characteristic 2, on the other hand, even the number of summands depends on the particular decomposition: the symmetric bilinear forms $x_{1} y_{1}+x_{2} y_{2}+x_{3} y_{3}$ and $x_{1} y_{2}+x_{2} y_{1}+x_{3} y_{3}$ are equivalent, but the form $x_{1} y_{2}+x_{2} y_{1}$ is indecomposable.

The rest of this section is devoted to the proof of Theorem 1 .

LEMMA 1. The radical of the endomorphism ring of a direct-sum-indecomposable representation is a nilpotent ideal and consists of all noninvertible endomorphisms.

ProOF. For every representation $B$, any endomorphism $f$ of $B$ satisfies Fitting's lemma: $B=\operatorname{Im}\left(f^{d}\right) \oplus \operatorname{Ker}\left(f^{d}\right)$, where $d=\operatorname{dim}(B)$, and $\operatorname{Im}(f)$ and $\operatorname{Ker}(f)$ are the restrictions of $B$ to the subspaces $\operatorname{Im}\left(f_{v}\right)$ and $\operatorname{Ker}\left(f_{v}\right)\left(v \in \mathscr{C}_{0}\right)$.

Suppose $B$ is direct-sum-indecomposable, and let $R$ be its set of noninvertible endomorphisms. Then $f^{d}=0$ for all $f \in R$. If $\operatorname{Im}(f)=\operatorname{Im}(f g)(f, g \in R)$, then $\operatorname{Im}(f)=\operatorname{Im}\left(f g^{d}\right)=0$, i.e., $f=0$. Consequently, $f_{1} \cdots f_{d}=0$ and $\left(f_{1}+f_{2}\right)^{d}=0$ for all $f_{i} \in R$, and $R$ is a nilpotent ideal of the endomorphism ring and coincides with the radical.

LEMMA 2. Let $A$ be a selfadjoint representation of the category $\mathscr{C}$, over a division ring $K$ of characteristic $\neq 2$, that is decomposable into a direct sum but not into an orthogonal one. Then $A$ is congruent to $B^{+}$, where $B \in \operatorname{ind}_{1}(\mathscr{C})$.

Proof. $1^{\circ}$. Let $f= \pm f^{0}$ be an endomorphism of $A$. By Fitting's lemma, $A=$ $\operatorname{Im}\left(f^{d}\right) \perp \operatorname{Ker}\left(f^{d}\right), d=\operatorname{dim}(A)$. But $A$ is indecomposable into an orthogonal sum. Therefore $f$ is either invertible or nilpotent.

$2^{\circ}$. Since $A$ is direct-sum-decomposable, there exists a nontrivial idempotent $e=e^{0} \in$ End $(A)$, the projection onto an indecomposable direct summand. From $1^{\circ}$ it follows that $e e^{0}$ is nilpotent. Consider the selfadjoint endomorphism $h=p\left(e e^{0}\right)$, where

$$
p(x)=1+a_{1} x+a_{2} x^{2}+\cdots
$$

is an infinite series with coefficients in the prime subfield of $K$ such that $p(x)^{2}=1-x$. The series exists, since the characteristic $\neq 2$.

Consider the idempotent $f=h^{-1} e h$. It satisfies

$$
f f^{0}=h^{-1} e h^{2} e^{0} h^{-1}=h^{-1} e\left(1-e e^{0}\right) e^{0} h^{-1}=h^{-1}\left(e e^{0}-e e^{0}\right) h^{-1}=0 .
$$


If we take a new $e$ equal to $f^{0}$, then $e^{0} e=0$ and for the new $f=h^{-1} e h$ we find, in addition to $f f^{0}=0$, that

$$
f^{0} f=h e^{0} h^{-2} e h=h e^{0}\left(1-e e^{0}\right)^{-1} e h=h e^{0}\left(1+e e^{0}\right) e h=0 .
$$

Consequently, $f+f^{0}$ is idempotent. By $1^{\circ}, f+f^{0}$ is a trivial idempotent. If $f+f^{0}=0$, then $f^{0}=-f$, a contradiction to $1^{\circ}$. Therefore $f+f^{0}=1$ and $A=D^{+}$, where the representation $D=\operatorname{Im}(f)$ is direct-sum-indecomposable (recall that $e$ was the projection onto an indecomposable direct summand).

The representation $D$ cannot be isomorphic to one that is selfadjoint. Indeed, suppose $\varphi: D \rightarrow B=B^{0}$ is an isomorphism. Then $\varphi \oplus\left(\varphi^{0}\right)^{-1}: D^{+} \rightarrow B^{+}$and $\psi: B^{+} \rightarrow B \perp$ $B^{-1}(-1 \in \operatorname{Aut}(B))$ are congruences, where

$$
\psi_{v}=\left(\begin{array}{rr}
1 / 2 & 1 \\
-1 / 2 & 1
\end{array}\right), \quad \psi_{v^{*}}=\psi_{v}^{*-1} \quad\left(v \in S_{0}\right)
$$

This contradicts the assumption that $A=D^{+}$is indecomposable into an orthogonal sum.

Consequently, the representation $D$ is isomorphic either to $B$ or to $B^{0}$, where $B \in$ ind ${ }_{1}(\mathscr{C})$. Then $D^{+}$is congruent either to $B^{+}$or to $\left(B^{0}\right)^{+}$. But $\left(B^{0}\right)^{+}$is congruent to $B^{+}$. Therefore $A=D^{+}$is congruent to $B^{+}$; and the lemma is proved.

REMARK. Over a ring of characteristic 2 the lemma is false, but a weaker version holds: if $A$ is the representation of the lemma, then $A \simeq B \oplus B^{0}$, where $B$ is indecomposable. Indeed, let $g=e+e^{0}+e e^{0} \in \operatorname{End}(A)$, where $e=e^{2}$ is the projection onto the direct summand $B$ of least dimension $\sum \operatorname{dim}\left(B_{u}\right)$. If $g$ is noninvertible, it is nilpotent (step $1^{\circ}$ in the proof of the lemma) and $h=1+g$ is invertible; which implies, since $e h=$ $e+e+e e^{0}+e e^{0}=0$, that $e=0$. Therefore $g$ must be invertible, and

$$
A=\left(e+e^{0}+e e^{0}\right) A=e A+e^{0} A \simeq B \oplus B^{0} .
$$

LEMMA 3. Let $A$ and $B$ be two selfadjoint representations $f=f^{0} \in \operatorname{Aut}(A), g=$ $g^{0} \in \operatorname{Aut}(B)$. Then $A^{f}$ is congruent to $B^{g}$ if and only if $g=h^{0} f h$ for some isomorphism $h: B \rightarrow A$.

ProOF. Suppose $\varphi: B^{g} \rightarrow A^{f}$ is a congruence. Define the isomorphism $h=$ $\left(\tilde{f}^{0}\right)^{-1} \varphi \tilde{g}^{0}: B \rightarrow A$, where $\tilde{f}$ and $\tilde{g}$ are the isomorphisms of form (2). Using the relations $f=\tilde{f} \tilde{f}^{0}, g=\tilde{g} \tilde{g}^{0}$, and $\varphi^{0} \varphi=1$, we find $h^{0} f h=g$. Conversely, if $g=h^{0} f h$, then $\varphi=\tilde{f}^{0} h\left(\tilde{g}^{0}\right)^{-1}: B^{g} \rightarrow A^{f}$ is a congruence.

LEMMA 4. Suppose a representation $A$ is selfadjoint and direct-sum-indecomposable. Then $A$ is congruent to a representation $B^{f}$, where $B \in \operatorname{ind}_{0}(\mathscr{C})$ and $f=f^{0} \in \operatorname{Aut}(B)$.

Proof. By definition of the set $\operatorname{ind}_{0}(\mathscr{C})$, there exists an isomorphism $h: B \rightarrow A$, $B \in \operatorname{ind}_{0}(\mathscr{C})$. By Lemma $3, B^{h^{0} h}$ is congruent to $B$.

LEMMA 5. Over a division ring $K$ of characteristic $\neq 2$, the representations $B^{f_{1}} \perp$ $\cdots \perp B^{f_{n}}$ and $B^{g_{1}} \perp \cdots \perp B^{g_{n}}\left(B \in \operatorname{ind}_{0}(\mathscr{C}) ; f_{i}=f_{i}^{0}, g_{i}=g_{i}^{0} \in \operatorname{Aut}(B)\right)$ are congruent if and only if, over the division ring $T(B)=\operatorname{End}(B) / R$, the Hermitian forms $\sum_{i} x_{i}^{0}\left(f_{i}+R\right) x_{i}$ and $\sum_{i} x_{i}^{0}\left(g_{i}+R\right) x_{i}$ are equivalent.

Proof. Obviously, $B^{f_{1}} \perp \cdots \perp B^{f_{n}}=D^{f}$, where $D=B \perp \cdots \perp B$ and $f=$ $\operatorname{diag}\left(f_{1}, \ldots, f_{n}\right)$. By Lemma $3, D^{f}$ is congruent to $D^{g}\left(g=\operatorname{diag}\left(g_{1}, \ldots, g_{n}\right)\right)$ if and only if $g=h^{0} f h$, where $h=\left(h_{i j}\right), h_{i j} \in \operatorname{End}(B)$. 
In particular, if $g_{i} \in f_{i}+R$, then $D^{g}$ is congruent to $D^{f}$. Indeed, let $h=p(r)$, where $p(x)$ is the series (4) and $r=\operatorname{diag}\left(r_{1}, \ldots, r_{n}\right), r_{i}=f_{i}^{-1}\left(f_{i}-g_{i}\right) \in R$ (by Lemma 1 the matrix $r$ is nilpotent). Then

$$
r_{i}^{0} f_{i}=\left(1-g_{i} f_{i}^{-1}\right) f_{i}=f_{i} r_{i}, \quad h^{0} f h=p\left(r^{0}\right) f h=f h^{2}=f(1-r)=g .
$$

Consequently, all the matrices in the set $\operatorname{diag}\left(f_{1}+R, \ldots, f_{n}+R\right)$ give congruent representations. Thus, $D^{g}$ is congruent to $D^{f}$ if and only if

$$
\operatorname{diag}\left(b_{1}, \ldots, b_{n}\right)=\left(c_{i j}\right)^{0} \operatorname{diag}\left(a_{1}, \ldots, a_{n}\right)\left(c_{i j}\right),
$$

where $a_{i}=f_{i}+R, b_{i}=g_{i}+R$, and $c_{i j}=h_{i j}+R$; i.e., if and only if the Hermitian forms $\sum x_{i}^{0} a_{i} x_{i}$ and $\sum x_{i}^{0} b_{i} x_{i}$ are equivalent over $T(B)$.

PROOF OF THEOREM 1. $1^{\circ}$. If

$$
M_{1} \oplus \cdots \oplus M_{t} \underset{g}{\stackrel{f}{\rightleftarrows}} N \oplus \cdots \oplus N
$$

are two homomorphisms of direct sums of indecomposable representations of the category $\mathscr{C}$, with $N$ nonisomorphic to any of the representations $M_{1}, \ldots, M_{t}$, then the endomorphism $h=f g$ is nilpotent. Indeed, $f=\left(f_{i j}\right), g=\left(g_{j k}\right)$, and $h=\left(h_{i k}\right)$, where $f_{i j}: M_{j} \rightarrow N, g_{j k}: N \rightarrow M_{j}$, and $h_{i k}=\sum_{j} f_{i j} g_{j k}: N \rightarrow N$. Since the set $R$ of noninvertible elements of the ring $\operatorname{End}(N)$ is a nilpotent ideal (Lemma 1), it suffices to show that $f_{i j} g_{j k} \in R$. Suppose that, on the contrary, $f_{i j} g_{j k}$ is invertible. Then so is $g_{j k} f_{i j}$ (since it is not nilpotent); and therefore $f_{i j}$ is an isomorphism, contradicting the assumption $M_{j} \not N$.

$2^{\circ}$. By Lemmas 2 and 4 , every selfadjoint representation is congruent to a representation $A$ of the form (3). Let

$$
C=C_{1}^{+} \perp \cdots \perp C_{k}^{+} \perp D_{1}^{\psi_{1}(x)} \perp \cdots \perp D_{l}^{\psi_{l}(x)}
$$

be a second representation of the same form, and $f: A \rightarrow C$ a congruence. Since the representations $A$ and $C$ are isomorphic, so are their indecomposable direct summands (the Krull-Schmidt theorem ([29], Chap. I, Theorem (3.6)) for the additive category $R(\mathscr{E})$ ). From this, in view of the isomorphism

$$
A \simeq \bigoplus_{i=1}^{m}\left(A_{i} \oplus A_{i}^{0}\right) \oplus \bigoplus_{j=1}^{n}\left(B_{j} \oplus \cdots \oplus B_{j}\right)
$$

(see (3) and (2)) and the definition of the sets $\operatorname{ind}_{0}(\mathscr{C})$ and $\operatorname{ind}_{1}(\mathscr{C})$, we find that $m=k$ and $n=l$, and, reindexing if necessary, $A_{i}=C_{i}, B_{j}=D_{j}, B_{j}^{\varphi_{j}(x)} \simeq B_{j}^{\psi_{j}(x)}$.

Write the congruence $f: A \rightarrow C$ as a matrix

$$
f=\left(\begin{array}{ll}
f_{11} & f_{12} \\
f_{21} & f_{22}
\end{array}\right): S \perp B_{n}^{\varphi_{n}(x)} \rightarrow T \perp B_{n}^{\psi_{n}(x)},
$$

where $S$ and $T$ are the sums (3) and (5) without the last summand. From $f^{0} f=1$ it follows that $f_{12}^{0} f_{12}+f_{22}^{0} f_{22}=1$. Since $f_{12}^{0} f_{12}$ is nilpotent (see $1^{\circ}$ ), we can define the homomorphism

$$
g=f_{22} p\left(f_{12}^{0} f_{12}\right)^{-1}: B_{n}^{\varphi_{n}(x)} \rightarrow B_{n}^{\psi_{n}(x)},
$$

where $p(x)$ is the series (4). Since

$$
g^{0} g=p\left(f_{12}^{0} f_{12}\right)^{-1}\left(1-f_{12}^{0} f_{12}\right) p\left(f_{12}^{0} f_{12}\right)^{-1}=1 \quad \text { and } \quad B_{n}^{\varphi_{n}(x)} \simeq B_{n}^{\psi_{n}(x)}
$$

$g$ is a congruence. By Lemma 5 , the Hermitian forms $\varphi_{n}(x)$ and $\psi_{n}(x)$ are equivalent. A similar argument gives equivalence of each of the forms $\varphi_{j}(x)$ and $\psi_{j}(x)(1 \leq j<n)$. This completes the proof of the theorem. 


\section{\$2. Applications to linear algebra}

In this section we apply Theorem 1 to some classical classification problems.

Let $\mathscr{C}$ be a linear category with involution over a field $P$. To specify the category $\mathscr{C}$, it suffices to list:

a) a set $S_{0} \subset \mathscr{C}_{0}$ of objects of the category such that $S_{0} \cup S_{0}^{*}=\mathscr{C}_{0}, S_{0} \cap S_{0}^{*}=\varnothing$,

b) a set $S_{1} \subset \mathscr{C}_{1}$ of generating morphisms, such that every morphism in the category is representable is a linear combination of products of morphisms in $S_{1} \cup S_{1}^{*} \cup\left\{1_{u}\right\}_{u \in \mathscr{C}_{0}}$, and

c) a set $S_{2}$ of defining relations for $\mathscr{C}$,

$$
\sum_{i} \alpha_{i 1} \cdots \alpha_{i t_{i}} a_{i}=0,
$$

where $a_{i} \in P$ and $\alpha_{i j} \in S_{1} \cup S_{1}^{*} \cup\left\{\mathbf{1}_{u}\right\}_{u \in \mathscr{K}_{0}}$, such that multiplication of morphisms in $\mathscr{C}$ is completely determined by the bilinearity property and the relations $S_{2} \cup S_{2}^{*}$, where $S_{2}^{*}$ consists of the adjoints of the relations in $S_{2}: \sum \alpha_{i t_{i}}^{*} \cdots \alpha_{i 1}^{*} \bar{a}_{i}=0$.

Let us agree, further, that the set $S_{1}$ is not to contain any morphisms of the form $\alpha: v^{*} \rightarrow u^{*}\left(u, v \in S_{0}\right)$ - since these can be replaced by the adjoint morphisms $\alpha^{*}: u \rightarrow v$.

If the sets $S_{0}$ and $S_{1}$ are finite, $S_{2}$ are also be taken to be finite. Such categories, called finitely generated, can be conveniently presented by graphs in the following two ways:

1. By a directed graph $\bar{S}$ with the set of vertices $\bar{S}_{0}=S_{0} \cup S_{0}^{*}$, the set of arrows $\bar{S}_{1}=S_{1} \cup S_{1}^{*}$, and the set of defining relations $\bar{S}_{2}=S_{2} \cup S_{2}^{*}$. Such a graph is called an involutive quiver in the category $\mathscr{C}$ (see [20]).

2. By a graph $S$ with the set of vertices $S_{0}$, the set of edges $S_{1}$, and the set of defining relations $S_{2}$; morphisms in $S_{1}$ of the form $\alpha: u \rightarrow v^{*}, \beta: u \rightarrow v$, and $\gamma: u^{*} \rightarrow v$ $\left(u, v \in S_{0}\right)$ and represented, respectively, by edges of the form $\alpha: u-v, \beta: u \rightarrow v$, and $\gamma: u \leftrightarrow v$. Such a graph, with nondirected, directed, and doubly directed edges, we call a discheme (directed scheme) in the category $\mathscr{C}$ (see [21]).

For example:

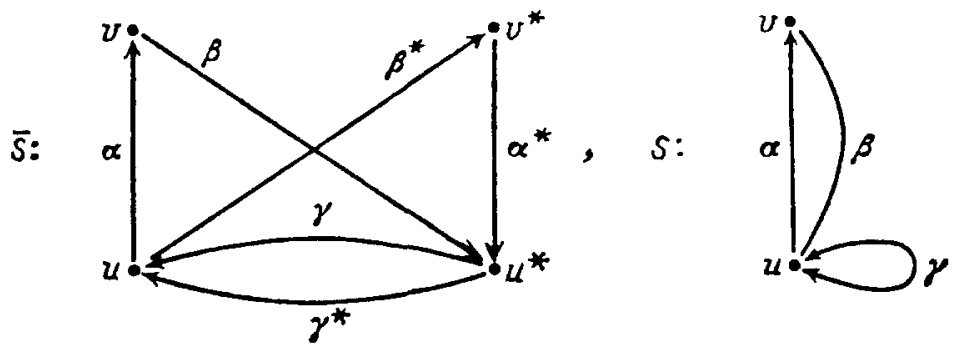

In what follows, a representation of the category $\mathscr{C}$ will be specified, not on the whole set $\mathscr{C}_{0} \cup \mathscr{C}_{1}$, but on the subset $\bar{S}_{0} \cup \bar{S}_{1}$ (being completely determined by its values on the subset); and we shall speak, correspondingly, not of a representation of the category $\mathscr{C}$, but of a representation of the quiver $\bar{S}$. Thus, a representation $A$ of the quiver $\bar{S}$ over a division ring $K$ is a set of finite-dimensional vector spaces $A_{v}\left(v \in \bar{S}_{0}\right)$ over $K$ and linear mappings $A_{\alpha}: A_{u} \rightarrow A_{v}\left(\bar{S}_{1} \ni \alpha: u \rightarrow v\right)$ satisfying the relations $\bar{S}_{2}$ (with the $\alpha \in \bar{S}_{1}$ replaced by the $A_{\alpha}$ ).

A selfadjoint representation is completely determined by its values on the set $S_{0} \cup S_{1}$, i.e., by a set of finite-dimensional vector spaces $A_{v}\left(v \in S_{0}\right)$ and linear mappings $A_{\alpha}$ 
$\left(\alpha \in S_{1}\right)$ of the form $A_{u} \rightarrow A_{v}^{*}$ for $\alpha: u-v, A_{u} \rightarrow A_{v}$ for $\alpha: u \rightarrow v$, and $A_{u}^{*} \rightarrow A_{v}$ for $\alpha: u \leftrightarrow v$, satisfying the relations $S_{2}$ (with $\alpha \in S_{1}$ and $\alpha^{*} \in S_{1}^{*}$ replaced by $A_{\alpha}$ and $A_{\alpha}^{*}$ ). Such a set will be called a representation $A$ of the discheme $S$ (see [21]).

A linear mapping $A: U \rightarrow V^{*}$ will be identified with the sesquilinear form $A: V \times U \rightarrow$ $K, A(v, u)=A(u)(v)$ (their matrices coincide, with the understanding that in the adjoint space we choose the adjoint basis). Recall that by a sesquilinear form is meant a mapping $A: V \times U \rightarrow K$ such that

$$
\begin{aligned}
A\left(v, u_{1}+u_{2}\right) & =A\left(v, u_{1}\right)+A\left(v, u_{2}\right), & & A(v, u a)=A(v, u) a, \\
A\left(v_{1}+v_{2}, u\right) & =A\left(v_{1}, u\right)+A\left(v_{2}, u\right), & A(v a, u) & =\vec{a} A(v, u) .
\end{aligned}
$$

With this identification, a representation $A$ of a discheme $S$ is a set of vector spaces $A_{v}$ $\left(v \in S_{0}\right)$, linear mappings, and sesquilinear forms $A_{\alpha}\left(\alpha \in S_{1}\right)$ of the form $A_{v} \times A_{u} \rightarrow K$ for $\alpha: u-v, A_{u} \rightarrow A_{v}$ for $\alpha: u \rightarrow v$, and $A_{v}^{*} \times A_{u}^{*} \rightarrow K$ for $\alpha: u \leftrightarrow v$ (in other words, the $A_{\alpha}$ are doubly covariant, mixed, or doubly contravariant tensors on the spaces $A_{u}$ and $A_{v}$ ). For two representations $A$ and $B$ of a discheme there is also a natural translation of the notions of congruence $f: A \rightarrow B$-a set of nonsingular linear mappings $f_{v}: A_{v} \rightarrow B_{v}\left(v \in S_{0}\right)$ taking the $A_{\alpha}$ into the $B_{\alpha}\left(\alpha \in S_{1}\right)$-and of orthogonal sum: $(A \perp B)_{x}=A_{x} \oplus B_{x}\left(x \in S_{0} \cup S_{1}\right)$.

EXAMPLE 1. The problems of classifying, up to congruence, the representations over a division ring $K$ of the dischemes

$$
\begin{aligned}
& \stackrel{v}{\bullet} \alpha \text {; } \\
& \alpha \bigcirc \stackrel{v}{\supset \beta}, \alpha=\varepsilon \alpha^{*}, \beta=\delta \beta^{*} \text {; } \\
& \alpha \int_{\gamma}^{\beta}, \beta=\alpha^{*} \beta \alpha, \beta=\varepsilon \beta^{*}, \quad \gamma \beta=l_{u}, \beta \gamma=1_{v^{*}} ; \\
& \alpha \mathrm{C}_{\gamma}^{\beta}, \beta \alpha=\alpha^{*} \beta, \beta=\varepsilon \beta^{*}, \gamma \beta=1_{v}, \beta \gamma=I_{v^{*}}
\end{aligned}
$$

(where $\varepsilon$ and $\delta$ are elements of the center of $K, \varepsilon \bar{\varepsilon}=\delta \bar{\delta}=1$ ), are the problems of classifying, respectively:

a) sesquilinear forms over $K$ (discheme (6));

b) pairs of forms, one of them $\varepsilon$-Hermitian, the other $\delta$-Hermitian (discheme (7)); and

c) isometric (8) and selfadjoint (9) operators in a space with nondegenerate $\varepsilon$-Hermitian form (an operator $A$ is isometric for a form $F(x, y)$ if $F(A x, A y)=F(x, y)$; it is selfadjoint if $F(x, A y)=F(A x, y))$.

EXAMPLE 2. The problem of classifying the representations of a group $G$ by isometries of a nondegenerate $\varepsilon$-Hermitian form is presented by the discheme (8), with the arrow $\alpha$ replaced by arrows $\alpha_{1}, \ldots, \alpha_{n}$ (these being generators of $G$ ), and the relation $\beta=\alpha^{*} \beta \alpha$ by the relations $\beta=\alpha_{i}^{*} \beta \alpha_{i}(1 \leq i \leq n)$ and the defining relations of $G$ (see [6], Chap. 7, no. 2.6). 
The rest of the paper has to do with the representations of the dischemes (6)-(9) over a field $K$ of characteristic $\neq 2$ (these, as well as the representations of some other dischemes, were announced in [24] and [25]). Without loss of generality, we can assume that $\varepsilon, \delta \in\{-1,1\}$ in the case of trivial involution on $K$, and $\varepsilon=\delta=1$ in the case of nontrivial involution (an $\varepsilon$-Hermitian form can be made Hermitian by multiplying by $1+\bar{\varepsilon}$ if $\varepsilon \neq-1$, and by $a-\bar{a} \neq 0$ if $\varepsilon=-1$ and the involution is nontrivial).

For any polynomial $f(x)=a_{0} x^{n}+a_{1} x^{n-1}+\cdots+a_{n} \in K[x]$ we define the polynomials

$$
\begin{gathered}
f^{\vee}(x)=\bar{a}_{n}^{-1}\left(\bar{a}_{n} x^{n}+\cdots+\bar{a}_{1} x+\bar{a}_{0}\right) \quad \text { if } \alpha_{n} \neq 0, \\
\bar{f}(x)=\bar{a}_{0} x^{n}+\bar{a}_{1} x^{n-1}+\cdots+\bar{a}_{n} .
\end{gathered}
$$

By the adjoint of the matrix $A=\left(a_{i j}\right)$ we mean the matrix $A^{*}=\left(\bar{a}_{j i}\right)$ (this being the matrix of the adjoint operator in the adjoint bases). For a Frobenius cell $\Phi$ we denote by $\chi_{\Phi}(x)$ and $p_{\Phi}(x)$ the characteristic polynomial and its irreducible divisor; and by $\hat{\Phi}, \Phi_{\varepsilon}$, and $\Phi_{(\varepsilon)}(\varepsilon= \pm 1 ; \varepsilon=1$ for nontrivial involution) fixed nonsingular matrices satisfying, respectively, the conditions

$$
\begin{aligned}
& \text { a) } \hat{\Phi}=\hat{\Phi}^{*} \Phi \\
& \text { b) } \Phi_{\varepsilon}=\Phi_{\varepsilon}^{*}, \Phi_{\varepsilon} \Phi=\varepsilon\left(\Phi_{\varepsilon} \Phi\right)^{*} \\
& \text { c) } \Phi_{(\varepsilon)}=\varepsilon \Phi_{(\varepsilon)}^{*}=\Phi^{*} \Phi_{(\varepsilon)} \Phi
\end{aligned}
$$

Existence conditions and explicit forms for these matrices will be established in $\S 3$. The coefficients of the characteristic polynomial of a Frobenius cell we place in the last column. The following lemma will be employed in the construction of the set $\operatorname{ind}_{0}(\bar{S})$.

LEMMA 6. If a representation $A$ of a quiver $\bar{S}$ is isomorphic to one that is selfadjoint, then there exist a selfadjoint representation $B$ and an isomorphism $h: A \rightarrow B$ such that $h_{v}=1$ for all $v \in S_{0}$.

Proof. Let $f: A \rightarrow C=C^{0}$ be an isomorphism. Define $B=B^{0}$ and a congruence $g: C \rightarrow B$ as follows:

$$
\begin{gathered}
g_{u}=f_{u}^{-1}, \quad g_{u^{*}}=f_{u}^{*}, \quad B_{u}=A_{u}, \\
B_{u^{*}}=A_{u}^{*} \quad\left(u \in S_{0}\right), \quad B_{\alpha}=g_{w} C_{\alpha} g_{v}^{-1} \quad(\alpha: v \rightarrow w) .
\end{gathered}
$$

Then $h=g f: A \rightarrow B$ is the desired isomorphism.

\subsection{Classification of sesquilinear forms.}

LEMMA 7. Let $p(x)=p^{\vee}(x)$ be an irreducible polynomial of degree $2 r$ or $2 r+1$. Then every stationary element of the field $K(\kappa)=K[x] / p(x)$ with involution $f(\kappa)^{0}=\bar{f}\left(\kappa^{-1}\right)$ is uniquely representable in the form $q(\kappa)$, where

$$
q(x)=\bar{a}_{r} x^{-r}+\cdots+a_{0}+\cdots+a_{r} x^{r}
$$

$\left(a_{0}=\bar{a}_{0}, a_{1}, \ldots, a_{r} \in K\right)$, and when $\operatorname{deg}(p(x))=2 r$ the following hold:

1) $a_{r}=0$ if the involution on $K$ is trivial.

2) $a_{r}=\bar{a}_{r}$ if the involution on $K$ is nontrivial and $p(0) \neq 1$.

3) $a_{r}=-\bar{a}_{r}$ if the involution on $K$ is nontrivial and $p(0)=1$.

PROOF. $1^{\circ}$. Suppose $\operatorname{deg}(p(x))=2 r+1$. The elements $\kappa^{-r}, \ldots, 1, \ldots, \kappa^{r}$ are linearly independent over $K$. Therefore all elements of the form $a_{-r} \kappa^{-r}+\cdots+a_{r} \kappa^{r}$ are distinct. They are stationary if and only if $a_{-i}=\bar{a}_{i}(0 \leq i \leq r)$.

$2^{\circ}$. Suppose $\operatorname{deg}(p(x))=2 r$ and the involution on $K$ is trivial. Then the stationary elements of the form $a_{r-1} \kappa^{-r+1}+\cdots+a_{0}+\cdots+a_{r-1} \kappa^{r-1}$ are distinct and form a vector 
space of dimension $r$ over $K$. But this is the dimension over $K$ of the whole stationary subfield of the field $K(\kappa)$, and therefore the subfield and the vector space coincide.

$3^{\circ}$. Suppose $\operatorname{deg}(p(x))=2 r$ and the involution on $K$ is nontrivial. The equality $p(x)=p^{\vee}(x)$ implies that $\alpha \bar{\alpha}=1$, where $\alpha=p(0)$. Putting $\delta=1+\bar{\alpha}$ if $\alpha \neq-1$, and $\delta=a-\bar{a}(a \neq \bar{a} \in K)$ if $\alpha=-1$, we find that $\delta \alpha=\bar{\delta}$. The function $\pi(x)=\delta x^{-r} p(x)$ has the form

$$
\pi(x)=c_{-r} x^{-r}+\cdots+c_{r} x^{r} \quad\left(c_{-i}=\bar{c}_{i}\right) .
$$

Using the equalities $c_{r}=\delta$ and $\delta \alpha=\bar{\delta}$, we find that $c_{r} \neq \bar{c}_{r}$ if $\alpha \neq 1$, and $c_{r} \neq-\bar{c}_{r}$ if $\alpha=1$.

Let $q(x)$ be a function of the form (13). If $q(\kappa)=0$, then $q(x)=a \pi(x), a=\bar{a} \in$ $K$, and in view of conditions 2) and 3) of the lemma this is possible only if $q(x)=$ 0 . Consequently, the stationary elements $q(\kappa)$ are distinct and form a vector space of dimension $2 r$ over the stationary subfield $K_{0}$ of $K$. But this is the dimension over $K_{0}$ of the whole stationary subfield of $K(\kappa)$. This completes the proof of the lemma.

We define the direct and skew sums of two matrices, as follows:

$$
A \oplus B=\left(\begin{array}{cc}
A & 0 \\
0 & B
\end{array}\right), \quad A \backslash B=\left(\begin{array}{cc}
0 & B \\
A & 0
\end{array}\right) .
$$

THEOREM 3. For any sesquilinear form on a finite-dimensional vector space over a field $K$ of characteristic $\neq 2$, there exists a basis in which the matrix of the form is a direct sum of matrices of the following types:

1) a singular Frobenius cell,

2) $A_{\Phi}=\Phi \backslash E$, where $\Phi$ is a nonsingular Frobenius cell for which $\hat{\Phi}$ does not exist; the cells $\Phi$ and $E$ have the same dimension, and

3) $\hat{\Phi} q(\Phi)$, where $q(x) \neq 0$ is of the form (13).

Furthermore, the summands are determined to the following extent:

Type 1)-uniquely.

Type 2)-up to replacement of the cell $\Phi$ by a cell $\Psi$, where $\chi_{\Psi}(x)=\chi_{\Phi}^{\vee}(x)$.

Type 3)-up to replacement of the whole group of summands $\bigoplus_{i} \hat{\Phi} q_{i}(\Phi)$ with the same cell $\Phi$ by the group $\bigoplus_{i} \hat{\Phi} q_{i}^{\prime}(\Phi)$, where the Hermitian forms $\sum_{i} q_{i}(\kappa) x_{i}^{0} x_{i}$ and $\sum_{i} q_{i}^{\prime}(\kappa) x_{i}^{0} x_{i}$ are equivalent over the field $K(\kappa)=K[x] / p_{\Phi}(x)$ with involution $f(\kappa)^{0}=$ $\bar{f}\left(\kappa^{-1}\right)$.

In particular, if $K$ is an algebraically closed field with trivial involution, then the summands of type 3) can be taken equal to $\hat{\Phi}$. If $K$ is an algebraically closed field with nontrivial involution, or a maximal ordered field, then the summands of type 3) can be taken equal to $\pm \hat{\Phi}$. In these cases the summands are then uniquely determined by the sesquilinear form.

ProOF. $1^{\circ}$. We describe ind $(\bar{S})$. The discheme $(6)$ corresponds to the quiver

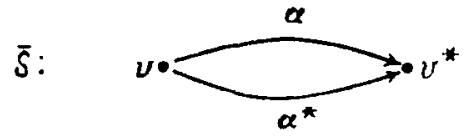

The representations of this quiver $\bar{S}$, as well as morphisms of the representations, will be specified by pairs of matrices. A representation is a matrix pair $\left(A_{\alpha}, A_{\alpha^{*}}\right)$ of the same dimension, with elements in $K$. A morphism $g:\left(A_{\alpha}, A_{\alpha^{*}}\right) \rightarrow\left(B_{\alpha}, B_{\alpha^{*}}\right)$ is a matrix pair $g=\left[G_{v}, G_{v^{*}}\right]$ (for morphisms we use square brackets) such that

$$
G_{v^{*}} A_{\alpha}=B_{\alpha} G_{v}, \quad G_{v^{*}} A_{\alpha^{*}}=B_{\alpha^{*}} G_{v}
$$


The adjoint of a representation is given by $\left(A_{\alpha}, A_{\alpha^{*}}\right)^{0}=\left(B_{\alpha}, B_{\alpha^{*}}\right)$, where $B_{\alpha}=A_{\alpha^{*}}^{*}$ and $B_{\alpha^{*}}=A_{\alpha}^{*}$.

As shown by Kronecker (the matrix pencil problem; see [30], Chap. XII), the set ind $(\bar{S})$ consists of the representations

$$
\left(N_{1}, N_{2}\right), \quad\left(N_{2}^{*}, N_{1}^{*}\right), \quad(\Phi, E), \quad\left(E, J_{n}\right),
$$

where $\Phi$ is an arbitrary, and $J_{n}$ a singular, Frobenius cell of dimension $n \times n$, and

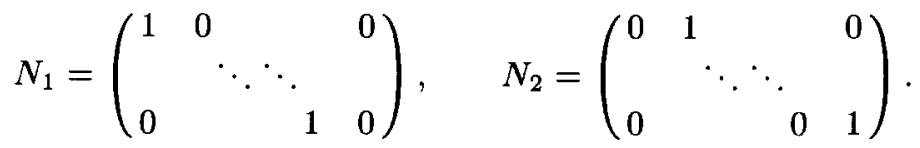

$2^{\circ}$. We describe $\operatorname{ind}_{0}(\bar{S})$ and $\operatorname{ind}_{1}(\bar{S})$. By $(15),(\Psi, E) \simeq(\Phi, E)^{0}=\left(E, \Phi^{*}\right)$ if and only if $\Psi$ is similar to $\Phi^{*-1}$; i.e., if and only if

$$
\chi_{\Psi}(x)=\left|x E-\Phi^{*-1}\right|=\left|-\Phi^{*-1}\right| \cdot x^{n} \cdot\left|x^{-1}-\Phi^{*}\right|=\chi_{\Phi}^{\vee}(x) .
$$

Suppose the representation $(\Phi, E)$ is isomorphic to a selfadjoint representation. By Lemma 6, there exists an isomorphism $h=[E, H]:(\Phi, E) \rightarrow\left(A, A^{*}\right)$. By (15), $A=H \Phi$ and $A^{*}=H$. Then $A=A^{*} \Phi$, and by (10) we can take

$$
h=\left[E, \hat{\Phi}^{*}\right]:(\Phi, E) \rightarrow\left(\hat{\Phi}, \hat{\Phi}^{*}\right) .
$$

Consequently, the set $\operatorname{ind}_{0}(\bar{S})$ consists of the representations $M_{\Phi}=\left(\hat{\Phi}, \hat{\Phi}^{*}\right)$. The set $\operatorname{ind}_{1}(\bar{S})$ consists of the representations $\left(N_{1}, N_{2}\right)$ and $(\Phi, E)$, where $\Phi$ is a Frobenius cell for which $\hat{\Phi}$ does not exist; and for nonsingular $\Phi$ the polynomial $\chi_{\Phi}(x)$ is determined up to replacement by $\chi_{\Phi}^{\vee}(x)$.

$3^{\circ}$. We describe the orbits of the representations in $\operatorname{ind}_{0}(\bar{S})$. Let $g=\left[G_{1}, G_{2}\right] \in$ $\operatorname{End}\left(M_{\Phi}\right)$ and $h$ be the isomorphism (18). Then $h^{-1} g h=\left[G_{1}, \hat{\Phi}^{*-1} G_{2} \hat{\Phi}^{*}\right]:(\Phi, E) \rightarrow$ $(\Phi, E)$; i.e., $G_{1}=\hat{\Phi}^{*-1} G_{2} \hat{\Phi}^{*}$ and $\Phi G_{1}=G_{1} \Phi$. Since a matrix that commutes with a Frobenius cell is a polynomial in this cell, we have

$$
\begin{aligned}
& G_{1}=f(\Phi) \quad(f(x) \in K[x]), \\
& G_{2}=\hat{\Phi}^{*} f(\Phi) \hat{\Phi}^{*-1}=f\left(\hat{\Phi}^{*} \Phi \hat{\Phi}^{*-1}\right)=f\left(\Phi^{*-1}\right) .
\end{aligned}
$$

Consequently, the ring $\operatorname{End}\left(M_{\Phi}\right)$ consists of matrix pairs $g_{f}=\left[f(\Phi), f\left(\Phi^{*-1}\right)\right], f(x) \in$ $K[x]$, with involution $g_{f}^{0}=\left[\vec{f}\left(\Phi^{-1}\right), \bar{f}\left(\Phi^{*}\right)\right]$. By Lemma 1 , its radical $R$ consists of the pairs $g_{f}$ for which $f(x) \in p_{\Phi}(x) K[x]$. Hence the field $T\left(M_{\Phi}\right)=\operatorname{End}\left(M_{\Phi}\right) / R$ can be identified with the field $K(\kappa)=K[x] / p_{\Phi}(x)$ with involution $f(\kappa)^{0}=\bar{f}\left(\kappa^{-1}\right)$.

Under this identification, a stationary element $q(\kappa) \neq 0$ of the field $K(\kappa)$ (where $q(x)$ is a function of form (13)) corresponds to the coset in the quotient ring $\operatorname{End}\left(M_{\Phi}\right) / R$ that contains the selfadjoint automorphism $\left[q(\Phi), q\left(\Phi^{*-1}\right)\right]$. By (2), the representations $M_{\Phi}^{q(\kappa)}=\left(\hat{\Phi}_{q}(\Phi), \hat{\Phi}^{*} q(\Phi)\right)$ constitute the orbit of the representation $M_{\Phi}$.

$4^{\circ}$. We now apply Theorems 1 and 2 . Each selfadjoint representation $\left(A, A^{*}\right)$ of the quiver (14) corresponds, in a one-to-one manner, to the representation of the discheme (6) given by the matrix $A$. In particular, we see from (1) that the representation $(A, B)^{+}$ of the quiver corresponds to the representation $A \backslash B^{*}$ of the discheme. From Theorem 1 and items $2^{\circ}$ and $3^{\circ}$ above, it follows that every representation of the discheme (6) is congruent to an orthogonal sum of representations of the form $N_{1} \backslash N_{2}^{*}, \Phi \backslash E$ (where $\hat{\Phi}$ does not exist), and $\hat{\Phi} f(\Phi)$. But the representations $N_{1} \backslash N_{2}^{*}$ and $J_{n} \backslash E$ are congruent to a singular Frobenius cell, since

a) $S^{*}\left(N_{1} \backslash N_{2}^{*}\right) S=J_{2 n-1}$, where $S=\left(s_{i j}\right), s_{\alpha, 2 \alpha-1}=s_{n+\beta, 2 \beta}=1(1 \leq \alpha \leq n, 1 \leq$ $\beta \leq n-1)$, and the remaining $s_{i j}$ are 0 ; and 
b) $S^{*}\left(J_{n} \backslash E\right) S=J_{2 n}$, where $S=\left(s_{i j}\right), s_{\alpha, 2 \alpha}=s_{n+\alpha, 2 \alpha-1}=1(1 \leq \alpha \leq n)$, and the remaining $s_{i j}$ are 0 .

This proves the first assertion of Theorem 3 (concerning existence of a basis). The remaining assertions follow from Theorems 1 and 2 .

REMARK. It can be shown that over an algebraically closed field of characteristic 2 , there exists for any bilinear form a basis in which its matrix is a direct sum

$$
\left(\Phi_{1} \backslash E\right) \oplus \cdots \oplus\left(\Phi_{r} \backslash E\right) \oplus \hat{\Psi}_{1} \oplus \cdots \oplus \hat{\Psi}_{t} \oplus F_{1} \oplus \cdots \oplus F_{s}
$$

where the $\Phi_{i}$ and $\Psi_{j}$ are nonsingular Jordan cells, $\Phi_{i} \neq \Psi_{j}$ for all $i$ and $j$, and the $F_{k}$ are singular Jordan cells. The direct sum is uniquely determined by the bilinear form up to permutation of the summands and replacement of the eigenvalue $\lambda$ in a cell $\Phi_{i}$ by $\lambda^{-1}$. The matrix $\hat{\Psi}$ exists if and only if the matrix $\Psi$ is of odd dimension with eigenvalue 1 .

\subsection{Classification of pairs of Hermitian forms.}

LEMMA 8. Let $K$ be a field with trivial involution, and suppose $A=\varepsilon A^{*}$ and $A \Phi=$ $\delta(A \Phi)^{*}$ for a nonsingular matrix $A$ and Frobenius cell $\Phi$. Then either $\varepsilon=1$ or $\delta=1$. If $\chi_{\Phi}(x)=x^{n}$, then $\varepsilon=1$ for $n$ odd and $\delta=1$ for $n$ even.

ProOF. Let $A=\left(a_{i j}\right)$, with dimension $n \times n$. Since multiplication by a Frobenius cell moves the columns of this matrix to the left, we have $A \Phi=\left(a_{i, j+1}\right)$ (the elements $a_{i, n+1}$ are defined by this equality). The relations $A=\varepsilon A^{*}$ and $A \Phi=\delta(A \Phi)^{*}$ can then be written

$$
a_{i j}=\varepsilon a_{j i}, \quad a_{i, j+1}=\delta a_{j, i+1} .
$$

Consequently, $a_{i j}=\varepsilon \delta a_{i-1, j+1}=(\varepsilon \delta)^{-i} b_{i+j}$, where $b_{2}, \ldots, b_{2 n} \in K$. Putting $i=j$ in (19), we find that $b_{2 i}=0$ if $\varepsilon \neq 1$, and $b_{2 i+1}=0$ if $\delta \neq 1$. Since $A \neq 0$, this implies either $\varepsilon=1$ or $\delta=1$. If $\chi_{\Phi}(x)=x^{n}$, then the formula $A \Phi=\left(b_{i+j+1}\right)$ implies $b_{n+2}=b_{n+3}=\cdots=0$; and since $A=\left(b_{i+j}\right)$ is nonsingular, this means $b_{n+1} \neq 0$, and therefore $\varepsilon=1$ for $n$ odd, $\delta=1$ for $n$ even.

For any matrices $A, B, C, D$ we define

$$
(A, B) \oplus(C, D)=(A \oplus C, B \oplus D), \quad(A, B) C=(A C, B C) .
$$

THEOREM 4. Let $F_{1}$ and $F_{2}$ be $\varepsilon$ - and $\delta$-Hermitian forms, respectively, in a finitedimensional vector space over a field $K$ of characteristic $\neq 2(\varepsilon= \pm 1, \delta= \pm 1, \varepsilon \geq \delta$, and $\varepsilon=\delta=1$ for nontrivial involution on $K$ ). Then there exists a basis in which the pair $\left(F_{1}, F_{2}\right)$ is given by a direct sum of matrix pairs of the following types:

1) $\left(N_{1} \backslash \varepsilon N_{1}^{*}, N_{2} \backslash \delta N_{2}^{*}\right)$, where $N_{1}$ and $N_{2}$ are as in (17).

2) $\left(E \backslash \varepsilon E, \Phi \backslash \delta \Phi^{*}\right)$, where $\Phi$ is a Frobenius cell, and $\Phi_{\delta}$ (see (11)) does not exist if $\varepsilon=1$.

3) $A_{\Phi}^{f(x)}=\left(\Phi_{\delta}, \Phi_{\delta} \Phi\right) f(\Phi)$, where $\varepsilon=1,0 \neq f(x)=\bar{f}(\delta x) \in K[x]$, and $\operatorname{deg}(f(x))<$ $\operatorname{deg}\left(p_{\Phi}(x)\right)$.

4) $\left(J \backslash \varepsilon J_{n}^{*}, E \backslash(-E)\right)$, where $\delta=-1, J_{n}$ is a singular Frobenius cell of dimension $n \times n$, and $n$ is odd if $\varepsilon=1$. 
5)

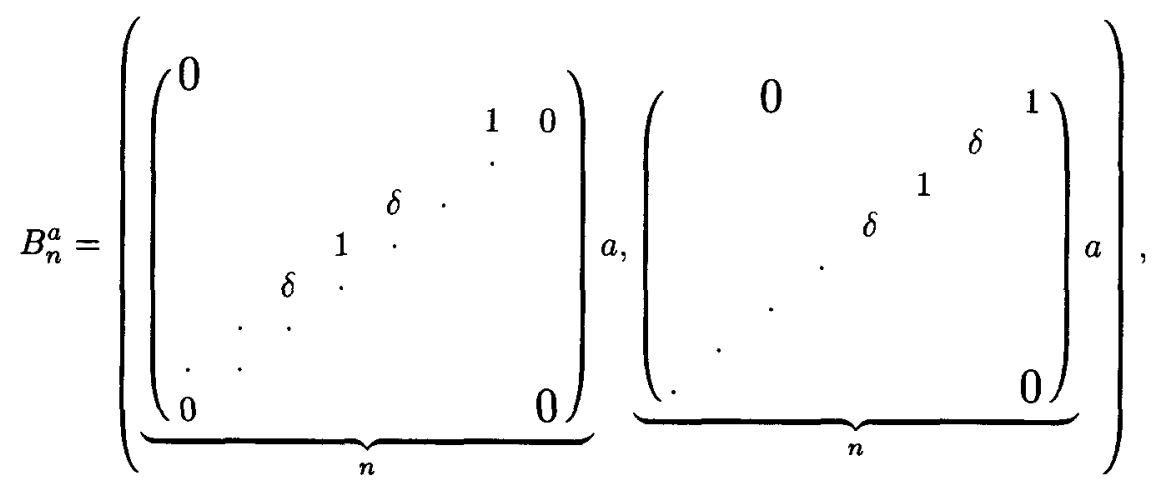

where $\varepsilon=1,0 \neq a=\bar{a} \in K$, and $n$ is even if $\delta=-1$.

The summands are determined to the following extent:

Type 1)-uniquely.

Type 2) -up to replacement of $\Phi$ by $\Psi$, where $\chi_{\Psi}(x)= \pm \bar{\chi}_{\Phi}(\varepsilon \delta \bar{x})$.

Type 3)-up to replacement of the whole group of summands $\bigoplus_{i} A_{\Phi}^{f_{i}(x)}$ with the same cell $\Phi$ by the group $\bigoplus_{i} A_{\Phi}^{g_{i}(x)}$, where the Hermitian forms $\sum_{i} f_{i}(\omega) x_{i}^{0} x_{i}$ and $\sum_{i} g_{i}(\omega) x_{i}^{0} x_{i}$ are equivalent over the field $K(\omega)=K[x] / p_{\Phi}(x)$ with involution $f(\omega)^{0}=\bar{f}(\delta \omega)$.

Type 4)-uniquely.

Type 5) - up to replacement of the whole group of summands $\bigoplus_{i} B_{n}^{a_{i}}$ with the same index $n$ by the group $\bigoplus_{i} B_{n}^{b_{i}}$, where the Hermitian forms $\sum_{i} a_{i} \bar{x}_{i} x_{i}$ and $\sum_{i} b_{i} \bar{x}_{i} x_{i}$ are equivalent over $K$.

ProOF. $1^{\circ}$. We describe ind $(\bar{S})$. The discheme (7) corresponds to the quiver

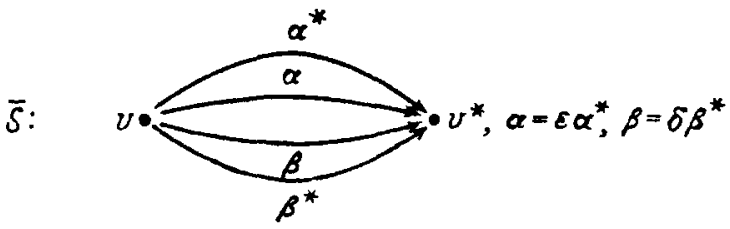

The representation of the quiver $\bar{S}$ will be specified by pairs of matrices $\left(A_{\alpha}, A_{\beta}\right)$ of the same dimension (and then $A_{\alpha^{*}}=\varepsilon A_{\alpha}$ and $A_{\beta^{*}}=\delta A_{\beta}$ ). The adjoint representation is given by $\left(A_{\alpha}, A_{\beta}\right)^{0}=\left(\varepsilon A_{\alpha}^{*}, \delta A_{\beta}^{*}\right)$. The set ind $(\bar{S})$ consists of the representations

$$
\left(N_{1}, N_{2}\right), \quad\left(N_{1}^{*}, N_{2}^{*}\right), \quad(E, \Phi), \quad\left(J_{n}, E\right)
$$

(which we prefer now to the set (16)).

$2^{\circ}$. We describe $\operatorname{ind}_{0}(\bar{S})$ and $\operatorname{ind}_{1}(\bar{S})$. It is obvious that $(E, \Psi) \simeq(E, \Phi)^{0}=\left(\varepsilon E, \delta \Phi^{*}\right)$ if and only if $\Psi$ is similar to $\varepsilon \delta \Phi^{*}$, i.e., if and only if $\chi_{\Psi}(x)= \pm \bar{\chi}_{\Phi}(\varepsilon \delta x)$.

Suppose $(E, \Phi)$ is isomorphic to a selfadjoint representation. By Lemma 6 , there exists an isomorphism $h=[E, H]:(E, \Phi) \rightarrow(A, B)=(A, B)^{0}$. Then $A=H, B=H \Phi$, $A=\varepsilon A^{*}$, and $B=\delta B^{*}$; i.e., $A=\varepsilon A^{*}$ and $A \Phi=\delta(A \Phi)^{*}$. Since $\varepsilon \geq \delta$, we have by Lemma 8 that $\varepsilon=1$, and by (11),

$$
h=\left[E, \Phi_{\delta}\right]:(E, \Phi) \rightarrow\left(\Phi_{\delta}, \Phi_{\delta} \Phi\right) .
$$


Similarly, if $\left(J_{n}, E\right) \simeq(A, B)=(A, B)^{0}$, then $B=\delta B^{*}$ and $B J_{n}=\varepsilon\left(B J_{n}\right)^{*}$; by Lemma $8, \varepsilon=1$, and $n$ is even if $\delta=-1$. It is easily verified that $\left(J_{n}, E\right) \simeq B_{n}$, where $B_{n}=B_{n}^{1}$ is of the form (20).

Consequently, the set ind $\operatorname{in}_{0}(\bar{S})$ is empty if $\varepsilon=-1$, and consists of the representations $A_{\Phi}=\left(\Phi_{\delta}, \Phi_{\delta} \Phi\right)$ and $B_{n}$ (where $n$ is even when $\delta=-1$ ) if $\varepsilon=1$. The set $\operatorname{ind}_{1}(\bar{S})$ consists of the following representations:

a) $\left(N_{1}, N_{2}\right)$.

b) $(E, \Phi)$, where $\Phi_{\delta}$ does not exist if $\varepsilon=1$, and $\chi_{\Phi}(x)$ is determined up to replacement $\bar{\chi}_{\Phi}(\varepsilon \delta x)$.

c) $\left(J_{n}, E\right)$, where $\delta=-1$, and $n$ is odd if $\varepsilon=1$.

$3^{\circ}$. We describe the orbits of the representations in $\operatorname{ind}_{0}(\bar{S})$. Let $g=\left[G_{1}, G_{2}\right] \in$ $\operatorname{End}\left(A_{\Phi}\right)$, and $h$ be the isomorphism (21). Then $h^{-1} g h=\left[G_{1}, \Phi_{\delta}^{-1} G_{2} \Phi_{\delta}\right]:(E, \Phi) \rightarrow$ $(E, \Phi)$; i.e., $G_{1}=\Phi_{\delta}^{-1} G_{2} \Phi$ and $\Phi G_{1}=G_{1} \Phi$. Since $G_{1}$ commutes with $\Phi$, we have $G_{1}=f(\Phi)(f(x) \in K[x]) ;$ and by $(11), G_{2}=\Phi_{\delta} f(\Phi) \Phi_{\delta}^{-1}=f\left(\Phi_{\delta} \Phi \Phi_{\delta}^{-1}\right)=f\left(\delta \Phi^{*}\right)$.

Consequently, the ring $\operatorname{End}\left(A_{\Phi}\right)$ consists of the matrix pairs $g_{f}=\left[f(\Phi), f\left(\delta \Phi^{*}\right)\right]$, $f(x) \in K[x]$, with involution $g_{f}^{0}=\left[\vec{f}(\delta \Phi), f(\Phi)^{*}\right]$. Hence the field $T\left(A_{\Phi}\right)=\operatorname{End}\left(A_{\Phi}\right) / R$ can be identified with the field $K(\omega)=K[x] / p_{\Phi}(x)$, with involution $f(\omega)=\bar{f}(\delta \omega)$. The set of representations $A_{\Phi}^{f(\omega)}=A_{\Phi} f(\Phi)$, where $0 \neq f(x)=\bar{f}(\delta x) \in K[x]$ and $\operatorname{deg}(f(x))<\operatorname{deg}\left(p_{\Phi}(x)\right)$, is the orbit of the representation $A_{\Phi}$.

Similarly, $T\left(B_{n}\right)$ can be identified with the field $K$, and the set of representations of the form $B_{n} a$, where $0 \neq a=\bar{a} \in K$ is the orbit of the representation $B_{n}$.

$4^{\circ}$. From $2^{\circ}, 3^{\circ},(1)$ and Theorem 1 , the proof of Theorem 4 now follows.

\subsection{Classification of isometric operators.}

THEOREM 5. Let $A$ be an isometric operator in a finite-dimensional vector space with nondegenerate $\varepsilon$-Hermitian form $F$ over a field $K$ of characteristic $\neq 2$. Then there exists a basis in which the pair $(A, F)$ is given by a direct sum of matrix pairs of the following types:

1) $\left(\Phi \oplus \Phi^{*-1}, E \backslash \varepsilon E\right)$, where $\Phi$ is a nonsingular Frobenius cell for which $\Phi_{(\varepsilon)}$ (see 12)) does not exist.

2) $A_{\Phi}^{q(x)}=\left(\Phi, \Phi_{(\varepsilon)} q(\Phi)\right)$, where $q(x) \neq 0$ is of form (13).

The summands are determined to the following extent:

Type 1)--up to replacement of $\Phi$ by $\Psi$, where $\chi \Psi(x)=\chi_{\Phi}^{\vee}(x)$.

Type 2)-up to replacement of the whole group of summands $\bigoplus_{i} A_{\Phi}^{q_{i}(x)}$ with the same cell $\Phi$ by the group $\bigoplus_{i} A_{\Phi}^{q_{i}(x)}$, where the Hermitian forms $\sum_{i} q_{i}(\kappa) x_{i}^{0} x_{i}$ and $\sum_{i} q_{i}^{\prime}(\kappa) x_{i}^{0} x_{i}$ are equivalent over the field $K(\kappa)=K[x] / p_{\Phi}(x)$ with involution $f(\kappa)^{0}=\bar{f}\left(\kappa^{-1}\right)$.

ProOF. $1^{\circ}$. We describe ind $(\bar{S})$. The discheme (8) corresponds to the quiver

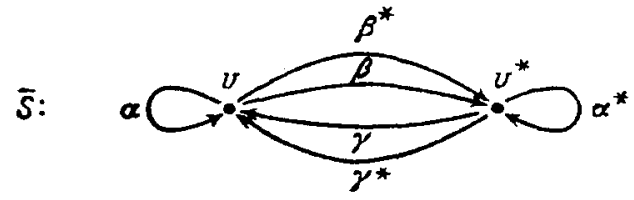

with relations $\beta=\alpha^{*} \beta \alpha, \beta=\varepsilon \beta^{*}, \gamma \beta=1_{v}, \beta \gamma=1_{v^{*}}, \gamma^{*} \beta^{*}=1_{v}$, and $\beta^{*} \gamma^{*}=$ $1_{v^{*}}$. The representations of the quiver will be specified by triples of square matrices 
$\left(A_{\alpha}, A_{\beta}, A_{\alpha^{*}}\right)$ of the same dimension, where $A_{\beta}$ is nonsingular and $A_{\beta}=A_{\alpha^{*}} A_{\beta} A_{\alpha}$ (and then $A_{\beta^{*}}=\varepsilon^{-1} A_{\beta}, A_{\gamma}=A_{\beta}^{-1}$, and $A_{\gamma^{*}}=\varepsilon A_{\beta}^{-1}$ ). The adjoint representation is given by $(A, B, C)^{0}=\left(C^{*}, \varepsilon B^{*}, A^{*}\right)$.

Every representation of the quiver is isomorphic to one of the form $\left(A, E, A^{-1}\right)$. The set ind $(\bar{S})$ consists of the representations $\left(\Phi, E, \Phi^{-1}\right)$, where $\Phi$ is a Frobenius cell.

$2^{\circ}$. We describe $\operatorname{ind}_{0}(\bar{S})$ and $\operatorname{ind}_{1}(\bar{S})$. It is obvious that

$$
\left(\Psi, E, \Psi^{-1}\right) \simeq\left(\Phi, E, \Phi^{-1}\right)^{0}=\left(\Phi^{*-1}, \varepsilon E, \Phi^{*}\right)
$$

if and only if $\Psi$ is similar to $\Phi^{*-1}$, i.e., if and only if $\chi_{\Psi}(x)=\chi_{\Phi}^{\vee}(x)$.

Suppose $\left(\Phi, E, \Phi^{-1}\right)$ is isomorphic to a selfadjoint representation. By Lemma 6 , there exists an isomorphism

$$
h=[E, H]:\left(\Phi, E, \Phi^{-1}\right) \rightarrow\left(A, B=\varepsilon B^{*}, A^{*}\right) .
$$

Then $A=\Phi, B=H, A^{*} H=H \Phi^{-1}$, and $B=\varepsilon B^{*}$; i.e., $A=\Phi$ and $B=\varepsilon B^{*}=\Phi^{*} B \Phi$. By $(12), h=\left[E, \Phi_{(\varepsilon)}\right]:\left(\Phi, E, \Phi^{-1}\right) \rightarrow\left(\Phi, \Phi_{(\varepsilon)}, \Phi^{*}\right)$.

Consequently, the set ind $\mathrm{d}_{0}(\bar{S})$ consists of the representations $A_{\Phi}=\left(\Phi, \Phi_{(\varepsilon)}, \Phi^{*}\right)$. The set $\operatorname{ind}_{1}(\bar{S})$ consists of the representations $\left(\Phi, E, \Phi^{-1}\right)$ where $\Phi$ is a Frobenius cell for which $\Phi_{(\varepsilon)}$ does not exist; and $\chi_{\Phi}(x)$ is determined up to replacement by $\chi_{\Phi}^{\vee}(x)$.

$3^{\circ}$. We describe the orbits of the representations in $\operatorname{ind}_{0}(\bar{S})$. Let $g=\left[G_{1}, G_{2}\right] \in$ $\operatorname{End}\left(A_{\Phi}\right)$. Then $\Phi G_{1}=G_{1} \Phi, \Phi_{(\varepsilon)} G_{1}=G_{2} \Phi_{(\varepsilon)}$, and $\Phi^{*} G_{2}=G_{2} \Phi^{*}$. Since $G_{1}$ commutes with the Frobenius cell, we have

$$
G_{1}=f(\Phi) \quad(f(x) \in K[x]), \quad \text { and } \quad G_{2}=\Phi_{(\varepsilon)} f(\Phi) \Phi_{(\varepsilon)}^{-1}=f\left(\Phi^{*-1}\right) .
$$

Consequently, the algebra $\operatorname{End}\left(A_{\Phi}\right)$ consists of the matrix pairs $\left[f(\Phi), f\left(\Phi^{*-1}\right)\right], f(x) \in$ $K[x]$, with involution $\left[f(\Phi), f\left(\Phi^{*-1}\right)\right]^{0}=\left[\bar{f}\left(\Phi^{-1}\right), f(\Phi)^{*}\right]$. The field $T\left(A_{\Phi}\right)$ can be identified with the field $K(\kappa)=K[x] / p_{\Phi}(x)$ with involution $f(\kappa)^{0}=\bar{f}\left(\kappa^{-1}\right)$. Let $q(\kappa)$ (where $q(x) \neq 0$ is of the form (13)) be a stationary element of this field. The representations $A_{\Phi}^{q(\kappa)}=\left(\Phi, \Phi_{(\varepsilon)} q(\Phi), \Phi^{*}\right)$ constitute the orbit of the representation $A_{\Phi}$.

$4^{\circ}$. From $2^{\circ}, 3^{\circ},(1)$, and Theorem 1 , the proof of Theorem 5 now follows.

\subsection{Classification of selfadjoint operators.}

THEOREM 6. Let $A$ be a selfadjoint operator in a finite-dimensional vector space with nondegenerate $\varepsilon$-Hermitian form $F$ over a field $K$ of characteristic $\neq 2(\varepsilon= \pm 1$; $\varepsilon=1$ for nontrivial involution on $K)$. Then there exists a basis in which the pair $(A, F)$ is given by a direct sum of matrix pairs of the following types:

1) $\left(\Phi \oplus \Phi^{*}, E \backslash \varepsilon E\right)$, where $\Phi$ is a Frobenius cell for which $\Phi_{1}$ (see (11)) does not exist if $\varepsilon=1$.

2) $A_{\Phi}^{f(x)}=\left(\Phi, \Phi_{1} f(\Phi)\right)$, where $\varepsilon=1,0 \neq f(x)=\bar{f}(x) \in K[x]$, and $\operatorname{deg}(f(x))<$ $\operatorname{deg}\left(p_{\Phi}(x)\right)$.

The summands are determined to the following extent:

Type 1)-up to replacement of $\Phi$ by $\Psi$, where $\chi_{\Psi}(x)=\bar{\chi}_{\Phi}(x)$.

Type 2)-up to replacement of the whole group of summands $\bigoplus_{i} A_{\Phi}^{f_{i}(x)}$ with the same cell $\Phi$ by the group $\bigoplus_{i} A_{\Phi}^{g_{i}(x)}$, where the Hermitian forms $\sum_{i} f_{i}(\omega) x_{i}^{0} x_{i}$ and $\sum_{i} g_{i}(\omega) x_{i}^{0} x_{i}$ are equivalent over the field $K(\omega)=K[x] / p_{\Phi}(x)$ with involution $f(\omega)^{0}=\bar{f}(\omega)$.

PROOF. $1^{\circ}$. We describe ind $(\bar{S})$. The discheme (9) corresponds to a quiver (22) with the relations $\beta \alpha=\alpha^{*} \beta, \beta=\varepsilon \beta^{*}, \gamma \beta=1_{v}, \beta \gamma=1_{v^{*}}, \gamma^{*} \beta^{*}=1_{v}$, and $\beta^{*} \gamma^{*}=1_{v^{*}}$. The representations of the quiver will be specified by triples of square matrices $\left(A_{\alpha}, A_{\beta}, A_{\alpha^{*}}\right)$ of the same dimension, where $A_{\beta}$ is nonsingular and $A_{\beta} A_{\alpha}=A_{\alpha^{*}} A_{\beta}$. The adjoint representation is given by $(A, B, C)^{0}=\left(C^{*}, \varepsilon B^{*}, A^{*}\right)$. 
Every representation of the quiver is isomorphic to one of the form $(A, E, A)$. The set ind $(\bar{S})$ consists of the representations $(\Phi, E, \Phi)$, where $\Phi$ is a Frobenius cell.

$2^{\circ}$. We describe $\operatorname{ind}_{0}(\bar{S})$ and $\operatorname{ind}_{1}(\bar{S})$. It is obvious that $(\Psi, E, \Psi) \simeq(\Phi, E, \Phi)^{0}=$ $\left(\Phi^{*}, \varepsilon E, \Phi^{*}\right)$ if and only if $\Psi$ is similar to $\Phi^{*}$, i.e., if and only if $\chi_{\Psi}(x)=\bar{\chi}_{\Phi}(x)$.

Suppose $(\Phi, E, \Phi)$ is isomorphic to a selfadjoint representation. By Lemma 6 , there exists an isomorphism $\left.h=[E, H]:(\Phi, E, \Phi) \rightarrow A, B=\varepsilon B^{*}, A^{*}\right)$. Then $A=\Phi, B=H$, $A^{*} H=H \Phi$, and $B=\varepsilon B^{*}$; i.e., $B=\varepsilon B^{*}$, and $B \Phi=\Phi^{*} B=\varepsilon(B \Phi)^{*}$. By Lemma 8 , $\varepsilon=1$, and we can take $B=\Phi_{1}$ (see (11)).

Consequently, the set $\operatorname{ind}_{0}(\bar{S})$ is empty if $\varepsilon=-1$, and consists of the representations $A_{\Phi}=\left(\Phi, \Phi_{1}, \Phi\right)$ if $\varepsilon=1$. The set ind ${ }_{1}(\bar{S})$ consists of the representations $(\Phi, E, \Phi)$, where $\Phi$ is a Frobenius cell, $\Phi_{1}$ does not exist if $\varepsilon=1$, and $\chi_{\Phi}(x)$ is determined up to replacement by $\bar{\chi}_{\Phi}(x)$.

$3^{\circ}$. We describe the orbits of the representations is ind $\mathrm{d}_{0}(\bar{S})$. Let $g=\left[G_{1}, G_{2}\right] \in$ $\operatorname{End}\left(A_{\Phi}\right)$. Then $\Phi G_{1}=G_{1} \Phi, \Phi_{1} G_{1}=G_{2} \Phi_{1}$, and $\Phi^{*} G_{2}=G_{2} \Phi^{*}$. Since $G_{1}$ commutes with the Frobenius cell, we have

$$
G_{1}=f(\Phi) \quad(f(x) \in K[x]), \quad G_{2}=\Phi_{1} f(\Phi) \Phi_{1}^{-1}=f\left(\Phi_{1} \Phi \Phi_{1}^{-1}=f\left(\Phi^{*}\right) .\right.
$$

Consequently, the algebra $\operatorname{End}\left(A_{\Phi}\right)$ consists of the matrix pairs $\left[f(\Phi), f\left(\Phi^{*}\right)\right], f(x) \in$ $K[x]$, with involution $\left[f(\Phi), f\left(\Phi^{*}\right)\right]^{0}=\left[\bar{f}(\Phi), f(\Phi)^{*}\right]$. The field $T\left(A_{\Phi}\right)$ can be identified with the field $K(\omega)=K[x] / p_{\Phi}(x)$ with involution $f(\omega)^{0}=\bar{f}(\omega)$. The set of representations $A_{\Phi}^{f(\omega)}=\left(\Phi, \Phi_{1} f(\Phi)\right)$, where $0 \neq f(x)=\bar{f}(x) \in K[x]$ and $\operatorname{deg}(f(x))<\operatorname{deg}\left(p_{\Phi}(x)\right)$, is the orbit of the representation $A_{\Phi}$.

$4^{\circ}$. From $2^{\circ}, 3^{\circ},(1)$, and Theorem 1 , the proof of Theorem 6 now follows.

\section{$\S 3$. The matrices $\hat{\Phi}, \Phi_{\varepsilon}$, and $\Phi_{(\varepsilon)}$}

In this section we obtain the existence conditions and forms for the matrices $\Phi, \Phi_{\varepsilon}$, and $\Phi_{(\varepsilon)}$ over a field $K$ of characteristic $\neq 2$ (see $(10)-(12)$ ); $\varepsilon= \pm 1$, and $\varepsilon=1$ for nontrivial involution on $K$.

In the case of nontrivial involution on $K$, we choose a fixed nonzero element

$$
k=-\bar{k} \neq 0
$$

we can take $k=a-\bar{a}, a \neq \bar{a} \in K$.

By $\Phi$ we denote a Frobenius cell of dimension $n \times n$, and by

$$
\begin{aligned}
& \chi(x)=p(x)^{s}=\alpha_{0} x^{n}+\alpha_{1} x^{n-1}+\cdots+\alpha_{n}, \\
& \mu(x)=p(x)^{s-1}=\beta_{0} x^{t}+\beta_{1} x^{t-1}+\cdots+\beta_{t}
\end{aligned}
$$

$\left(\alpha_{0}=\beta_{0}=1\right)$ we denote the characteristic polynomial of $\Phi$ and its maximal divisor.

Let $f(x)=\gamma_{0} x^{m}+\gamma_{1} x^{m-1}+\cdots+\gamma_{m} \in K[x]$. A sequence $\left(a_{q}, a_{q+1}, \ldots, a_{r}\right)$ of elements of $K$ will be called $f$-recurrent if

$$
\gamma_{0} a_{l+m}+\gamma_{1} a_{l+m-1}+\cdots+\gamma_{m} a_{l}=0
$$

$(q \leq l \leq r-m)$; the sequence is completely determined, assuming $\gamma_{0} \neq 0 \neq \gamma_{m}$, by any fragment of length $m$. The sequence will be called strictly $\chi$-recurrent if it is $\chi$-recurrent but not $\mu$-recurrent (see (24) and (25)).

LEMMA 9. The following two conditions on a matrix $A$ are equivalent:

a) $A=\Phi^{*} A \Phi$, and $A$ is nonsingular. 
b) $A=\left(a_{j-1}\right)$, where the sequence $\left(a_{1-n}, \ldots, a_{n-1}\right)$ is strictly $\chi$-recurrent, with $\chi(x)=\chi^{\vee}(x)$.

ProOF. a) $\Rightarrow$ b). Suppose the matrix $A=\left(a_{i j}\right)$ satisfies condition a). Then $A \Phi^{-1} A^{-1}=\Phi^{*}$, and

$$
\chi(x)=\left|x E-\Phi^{*-1}\right|=\left|-\Phi^{*-1}\right| \cdot x^{n} \cdot\left|x^{-1} E-\Phi^{*}\right|=\chi^{\vee}(x) .
$$

Since $\Phi^{*} A \Phi=\Phi^{*}\left(a_{i, j+1}\right)=\left(a_{i+1, j+1}\right)$ (the elements $a_{i, n+1}$ and $a_{n+1, j}$ are defined by this equality), we have $a_{i j}=a_{i+1, j+1}$, so that the matrix elements depend only on the difference of the indices; i.e., $A=\left(a_{j-i}\right)$. That the sequence $\left(a_{1-n}, \ldots, a_{n-1}\right)$ is $\chi$-recurrent follows from the equality $A \Phi=\left(a_{j-i+1}\right)$. Furthermore, the recurrence is strict; otherwise, we should have $\left(0, \ldots, 0, \beta_{0}, \ldots, \beta_{t}\right) A=0$ (see (25)), contradicting the assumption that $A$ is nonsingular.

b) $\Rightarrow$ a). Suppose b) is satisfied. Then $\Phi^{*} A \Phi=\Phi^{*}\left(a_{j-i+1}\right)=\left(a_{j-i}\right)=A$. We show now that $A$ is nonsingular. Suppose that, on the contrary, its rows $v \Phi^{n-1}$, $v \Phi^{n-2}, \ldots, v=\left(a_{1-n}, \ldots, a_{0}\right)$, are linearly dependent. Then $v f(\Phi)=0$ for some polynomial $f(x) \neq 0$ of degree less than $n$. Since $v \chi(\Phi)=0$, we have $v p(\Phi)^{r}=0$, where $p(x)^{r}$ is the greatest common divisor of the polynomials $f(x)$ and $\chi(x)$. But then $v \Phi^{i} \mu(\Phi)=\left(0, \ldots, 0, \beta_{0}, \ldots, \beta_{t}, 0, \ldots, 0\right) A=0(0 \leq i<n-t$; see (25)); and therefore the sequence $\left(a_{1-n}, \ldots, a_{n-1}\right)$ is $\mu$-recurrent, contradicting condition $\left.\mathrm{b}\right)$.

THEOREM 7. Existence conditions for the $n \times n$ matrix $\hat{\Phi}$ are:

(A1) $\chi(x)=\chi^{\vee}(x)$.

(A2) $p(x) \neq x+(-1)^{n-1}$ in the case of trivial involution.

With these conditions satisfied, we can take

$$
\hat{\Phi}=\left(a_{j-i}\right),
$$

where the sequence $\left(a_{1-n}, \ldots, a_{n-1}\right)$ is $\chi$-recurrent and is defined by the fragment

$$
\left(a_{-m}, \ldots, a_{m-1}\right)=(\bar{a}, 0, \ldots, 0, a)
$$

of length either $n$ or $n+1$, in which

a) $a=1$ if $n=2 m$, except for the case $p(x)=x+\alpha, \alpha^{n-1}=-1$;

b) $a=k$ (see (23)) if $n=2 m, p(x)=x+\alpha, \alpha^{n-1}=-1$, and also if $n=2 m-1$, $p(x)=x+1$

c) $a=\chi(-1)$ if $n=2 m-1, p(x) \neq x+1$.

ProOF. $1^{\circ}$. If the matrix $A=\hat{\Phi}$ exists, then conditions (A1) and (A2) must be satisfied. Indeed, in view of the relations

$$
A=A^{*} \Phi=\Phi^{*} A \Phi
$$

(see (10)) and Lemma 9, condition (A1) is satisfied, and the elements of the matrix $\left(a_{j-i}\right)=A=A^{*} \Phi=\left(\bar{a}_{i-j-1}\right)$ form a strictly $\chi$-recurrent sequence

$$
\left(a_{1-n}, \ldots, a_{n-1}\right)=\left(\bar{a}_{n-2}, \ldots, \bar{a}_{0}, a_{0}, \ldots, a_{n-1}\right) .
$$

This sequence is completely determined by the fragment

$$
\left(\bar{a}_{m-1}, \ldots, \bar{a}_{0}, a_{0}, \ldots, a_{m-1}\right)
$$

of length $2 m$, equal either to $n$ or to $n+1$.

Now suppose condition (A2) is not satisfied; i.e., that the involution is trivial and $p(x)=x+(-1)^{n-1}$. Then the vector $(28)$ is $\mu(x)=\left(x+(-1)^{n-1}\right)^{n-1}$-recurrent. For $n=2 m$ this is obvious; and for $n=2 m-1$ it follows from the property

$$
\alpha_{i}=\beta_{i}+\beta_{i-1}=\beta_{i}+\beta_{n-i}, \quad 0<i<n,
$$


of the binomial coefficients $\alpha_{i}$ and $\beta_{i}$ (see (24) and (25)), since

$$
\begin{aligned}
& 2\left[\beta_{0} a_{m-1}+\beta_{1} a_{m-2}+\cdots+\beta_{n-2} a_{m-3}+\beta_{n-1} a_{m-2}\right] \\
& =\left(\beta_{0}+0\right) a_{m-1}+\left(\beta_{1}+\beta_{n-1}\right) a_{m-2}+\left(\beta_{2}+\beta_{n-2}\right) a_{m-3} \\
& \quad+\cdots+\left(\beta_{n-1}+\beta_{1}\right) a_{m-2}+\left(0+\beta_{0}\right) a_{m-1} \\
& =\alpha_{0} a_{m-1}+\alpha_{1} a_{m-2}+\cdots+\alpha_{n} a_{m-1}=0
\end{aligned}
$$

in view of the $\chi$-recurrence of (28). But then its $\mu$-recurrent extension coincides with (27), contradicting the strict $\chi$-recurrence of (27).

$2^{\circ}$. If conditions (A1) and (A2) are satisfied, then the matrix $\hat{\Phi}$ exists. Indeed, let us verify that the vector (26) is strictly $\chi$-recurrent.

1) Suppose $n=2 m$. Since (26) is of length $n$, it suffices to verify that it is not $\mu$ recurrent. If $\operatorname{deg}(\mu(x))<n-1$, this is obvious. If $\operatorname{deg}(\mu(x))=n-1$, then the polynomial $\mu(x)$ is of the form $(x+\alpha)^{n-1}$, and therefore $a+\beta_{n-1} \bar{a}=a+\alpha^{n-1} \bar{a} \neq 0$.

2) Suppose $n=2 m-1$. Since (26) is of length $n+1$, it suffices to verify that it is $\chi$-recurrent, i.e., that $a+\alpha_{n} \bar{a}=0$ (see (24)). Condition (A1) implies that $\alpha_{n}=\bar{\alpha}_{n}^{-1}$, and so, since $\chi^{\vee}(x)=\bar{\alpha}_{n}^{-1} x^{n} \bar{\chi}\left(x^{-1}\right)$, that $\chi(-1)=-\alpha_{n} \overline{\chi(-1)}$. If $\chi(-1)=0$, then $\chi(x)=(x+1)^{n}, k+\alpha_{n} \bar{k}=0$.

Thus, the vector (26) is strictly $\chi$-recurrent, and its $\chi$-recurrent extension has, in view of (A1), the form (27). Consequently, $A=\left(a_{j-i}\right)=A^{*} \Phi$. By Lemma 9 , the matrix $A$ is nonsingular, and it can be taken to be $\hat{\Phi}$.

THEOREM 8. Existence conditions for the $n \times n$ matrix $\Phi_{\varepsilon}$ are:

(B1) $\chi(x)=\varepsilon^{n} \bar{\chi}(\varepsilon x)$.

(B2) $\chi(x) \notin\left\{x^{2}, x^{4}, x^{6}, \ldots\right\}$ if $\varepsilon=-1$.

With these conditions satisfied, we can take $\Phi_{\varepsilon}=\left(\varepsilon^{i} a_{i+j}\right)$, where $\left(a_{2}, a_{3}, \ldots, a_{2 n}\right)$ is a $\chi$-recurrent sequence defined by the fragment

a) $\left(a_{2}, \ldots, a_{n+1}\right)=(1,0, \ldots, 0)$ if $\Phi$ is nonsingular;

b) $\left(a_{2}, \ldots, a_{n+1}\right)=(0, \ldots, 0,1)$ if $\Phi$ is singular.

ProOF. $1^{\circ}$. Suppose $\Phi_{\varepsilon}$ exists. Then $\Phi=\Phi_{\varepsilon}^{-1}\left(\varepsilon \Phi^{*}\right) \Phi_{\varepsilon}$ (see (11)), and this gives condition (B1): $\chi(x)=\left|x E-\varepsilon \Phi^{*}\right|=\varepsilon^{n} \bar{\chi}(\varepsilon x)$. Condition (B2) follows from (11) and Lemma 8.

$2^{\circ}$. Suppose conditions (B1) and (B2) are satisfied. The matrix $\Phi_{\varepsilon}=\left(\varepsilon^{i} a_{i+j}\right)$ defined in the statement of Theorem 8 is nonsingular. Let us verify that it satisfies (11).

If $\Phi$ is singular, this is obvious.

Suppose $\Phi$ is nonsingular. Then the $\chi$-recurrence of the sequence $\left(a_{2}, a_{3}, \ldots, a_{2 n}\right)$ implies that $\Phi_{\epsilon} \Phi=\left(\varepsilon^{i} a_{i+j+1}\right)$; and so relations (11) can be written in the form

$$
\varepsilon^{i} a_{i+j}=\varepsilon^{j} \bar{a}_{j+i}, \quad \varepsilon^{i} a_{i+j+1}=\varepsilon \varepsilon^{j} \bar{a}_{j+i+1},
$$

i.e.,

$$
a_{t}=\varepsilon^{t} \bar{a}_{t}, \quad 2 \leq t \leq 2 n .
$$

We argue now by induction. Relation (30) certainly holds for $t \leq n+1$ (see a) and b) in the statement of the theorem). Assuming it holds for $t<n+l(l \geq 2)$, we must verify it for $t=n+l$. And indeed, using the $\chi$-recurrence of the sequence $\left(a_{2}, \ldots, a_{2 n}\right)$ and equalities (24) and (B1), we find that

$$
\begin{aligned}
a_{n+l} & =-\alpha_{1} a_{n+l-1}-\cdots-\alpha_{n} a_{l} \\
& =-\varepsilon \bar{\alpha}_{1} \varepsilon^{n+l-1} \bar{a}_{n+l-1}-\cdots-\varepsilon^{n} \bar{\alpha}_{n} \varepsilon^{l} \bar{a}_{l}=\varepsilon^{n+l} \bar{a}_{n+l} .
\end{aligned}
$$


THEOREM 9. Existence conditions for the $n \times n$ matrix $\Phi_{(\varepsilon)}$ are:

(C1) $\chi(x)=\chi^{\vee}(x)$.

(C2) If the involution on $K$ is trivial and $\varepsilon=(-1)^{n}$, then $\operatorname{deg}(p(x))>1$ (see (24)).

With these conditions satisfied, we can take $\Phi_{(\varepsilon)}=\left(a_{j-i}\right)$, where $\left(a_{1-n}, \ldots, a_{n-1}\right)$ is the $\chi$-recurrent sequence defined by the fragment $v=\left(a_{-m}, \ldots, a_{m}\right)$, of length either $n$ or $n+1$, equal to

a) $\left(\varepsilon \bar{\alpha}_{n}-1,0, \ldots, 0, \alpha_{n}-\varepsilon\right)$ if $n=2 m, \alpha_{n} \neq \varepsilon($ see (24));

b) $\left(\alpha_{1},-1,0, \ldots, 0,-1, \alpha_{1}\right)\left(v=\left(\alpha_{1},-2, \alpha_{1}\right)\right.$ for $\left.n=2\right)$ if $n=2 m, \varepsilon=1$, and the involution on $K$ is trivial;

c) $(-k, 0, \ldots, 0, k)($ see $(23))$ if $n=2 m, \alpha_{n}=1$, and the involution is nontrivial, and also if $n=2 m+1, p(x)=x+\alpha, \alpha^{n-1}=-1$;

d) $(\varepsilon, 0, \ldots, 0,1)$ if $n=2 m+1$, in any other case besides $p(x)=x+\alpha, \alpha^{n-1}=-1$.

ProOF. $1^{\circ}$. If the matrix $A=\Phi_{(\varepsilon)}$ exists, then conditions (C1) and (C2) are satisfied. Indeed, in view of the relations (12) and Lemma 9, condition (C1) is satisfied, and the elements of the matrix $A=\left(a_{j-i}\right)=\varepsilon A^{*}$ form a strictly $\chi$-recurrent sequence

$$
\left(a_{1-n}, \ldots, a_{n-1}\right)=\left(\varepsilon \bar{a}_{n-1}, \ldots, \varepsilon \bar{a}_{0}=a_{0}, \ldots, a_{n-1}\right) .
$$

Suppose condition (C2) is not satisfied. By (C1), $p(x)=p^{\vee}(x)=x \pm 1$, and the fragment $\left(\varepsilon a_{m}, \ldots, a_{m}\right)$, of length either $n$ or $n+1$, of the vector (31) is $\mu$-recurrent. This is obvious if $n=2 m+1$, since $\varepsilon=-1$; and if $n=2 m$, it follows from (29) as applied to the fragment (replace $m$ in (29) by $m+1$ ). But then the vector (31) is also $\mu$-recurrent, and we have a contradiction.

$2^{\circ}$. If conditions $(\mathrm{C} 1)$ and $(\mathrm{C} 2)$ are satisfied, then $\Phi_{(\varepsilon)}$ exists. To show this, let us verify that the vector $v$ of Theorem 9 is strictly $\chi$-recurrent and of the form $\left(\varepsilon \bar{a}_{m}, \ldots, \varepsilon \bar{a}_{0}=\right.$ $\left.a_{0}, \ldots, a_{m}\right)$.

1) The vector to a) is $\chi$-recurrent, since its length is $n+1$ and, by (C1), $\alpha_{n} \bar{\alpha}_{n}=1$.

2) The vector in b) is $\chi$-recurrent, since for trivial involution conditions (C1) and (C2) imply $\chi(1)=\alpha_{n}^{-1} \chi(1) \neq 0, \alpha_{n}=1, \alpha_{n-1}=\alpha_{1}$. The vector is not $\mu$-recurrent, since $t \leq n-2$ (by (25) and (C2)) and $\beta_{t}=1$ (by the equality $p(x)=p^{\vee}(x)$ and (C2)).

3) If $n=2 m+1, p(x)=x+\alpha$, and $\alpha^{n-1}=-1$ (see c)), then the involution is nontrivial: otherwise $p(x)=p^{\vee}(x)=x \pm 1$, contradicting the equality $\alpha^{n-1}=-1$.

4) The vector in d) is not $\mu$-recurrent, in view of (C2).

Now let (31) be the $\chi$-recurrent extension of the vector $v$. Then the matrix $A=\left(a_{j-i}\right)$ is equal to $\varepsilon A^{*}$, and by Lemma 9 it can be taken for $\Phi_{(\varepsilon)}$.

Received 10/NOV/85

\section{BIBLIOGRAPHY}

1. Peter Gabriel, Unzerlegbare Darstellungen. I, Manuscripta Math. 6 (1972), 71-103, 309.

2. I. N. Bernsteǐn, I. M. Gel'fand, and V. A. Ponomarev, Coxeter functors and Gabriel's theorem, Uspekhi Mat. Nauk 28 (1973), no. 2 (170), 19-33; English transl. in Russian Math. Surveys 28 (1973).

3. L. A. Nazarova, Representations of quivers of infinite type, Izv. Akad. Nauk SSSR Ser. Mat. 37 (1973), 752-791; English transl. in Math. USSR Izv. 7 (1973).

4. Claus Michael Ringel, Tame algebras and integral quadratic forms, Lecture Notes in Math., vol. 1099, Springer-Verlag, 1984.

5. O. T. O'Meara, Introduction to quadratic forms, Springer-Verlag, 1971.

6. Winfried Scharlau, Quadratic and Hermitian forms, Springer-Verlag, 1985.

7. Peter Gabriel, Appendix: degenerate bilinear forms, J. Algebra 31 (1974), 67-72.

8. Carl Riehm, The equivalence of bilinear forms, J. Algebra 31 (1974), 45-66.

9. C. Riehm and M. Shrader-Frechette, The equivalence of sesquilinear forms, J. Algebra 42 (1976), 495-530. 
10. Rudolf Scharlau, Zur Klassifikation von Bilinearformen und von Isometrien über Körpern, Math. Z. 178 (1981), 359-373.

11. I. M. Yaglom, Quadratic and skew-symmetric bilinear forms in a real symplectic space, Trudy Sem. Vektor. Tenzor. Analiz. 8 (1950), 364-381. (Russian)

12. Rudolf Scharlau, Paare alternierender Formen, Math. Z. 147 (1976), 13-19.

13. William C. Waterhouse, Pairs of quadratic forms, Invent. Math. 37 (1976), no. 2, 157-164.

14. Frank Uhlig, A recurring theorem about pairs of quadratic forms and extensions: a survey, Linear Algebra Appl. 25 (1979), 219-237.

15. _ A rational canonical pair form for a pair of symmetric matrices over an arbitrary field $F$ with char $F \neq 2$ and applications to finest simultaneous block diagonalizations, Linear and Multilinear Algebra 8 $(1979 / 80), 41-67$.

16. John Milnor, On isometries of inner product spaces, Invent. Math. 8 (1969), 83-97.

17. Bertram Huppert, Isometrien von Vektorräumen. I, Arch. Math. (Basel) 35 (1980), 164-176.

18. _ Isometrien von Vektorräumen. II, Math. Z. 175 (1980), 5-20.

19. D. W. Lewis, The isometry classification of Hermitian forms over division algebras, Linear Algebra Appl. 43 (1982), 245-272.

20. A. V. Roĭter, BOC's with involution, Representations and Quadratic Forms (Yu. A. Mitropol'skii, editor), Inst. Mat. Akad. Nauk Ukrain. SSR, Kiev, 1979, pp. 124-126. (Russian)

21. V. V. Sergel̆chuk, Representations of simple involutive quivers, Representations and Quadratic Forms (Yu. A. Mitropol'skiü, editor), Inst. Mat. Akad. Nauk Ukrain. SSR, Kiev, 1979, pp. 127-148. (Russian)

22. S. A. Kruglyak, Representations of involutive quivers, Preprint, Inst. Mat. Akad. Nauk Ukrain. SSR, Kiev, 1984=Manuscript No. 7266-84, deposited at VINITI, 1984. (Russian) R. Zh. Mat. 1985, $2 A 367$.

23. H.-G. Quebbemann, W. Scharlau, and M. Schulte, Quadratic and Hermitian forms in additive and abelian categories, J. Algebra 59 (1979), 264-289.

24. V. V. Sergeichuk, Representations of dischemes, Linear Algebra and the Theory of Representations (Yu. A. Mitropol'skir, editor), Inst. Mat. Akad. Nauk Ukrain. SSR, Kiev, 1983, pp. 110-134. (Russian)

25. ___ Classification problems for systems of linear mappings and sesquilinear forms, Preprint, Kiev. Gos. Univ., Kiev, 1983 =Manuscript No. 196 Uk-D84, depoisited at the Ukrainian NIINTI, 1984. (Russian) R. Zh. Mat. 1984, 7 A331.

26. N. Bourbaki, Algèbre, Chaps. 6, 7, Actualités Sci. Indust., no. 1179, Hermann, Paris, 1952.

27. __ Algèbre, Chaps. 8, 9, Actualités Sci. Indust., nos. 1261, 1272, Hermann, Paris, $1958,1959$.

28. Jean A. Dieudonné, La géométrie des groupes classiques, 3me éd., Springer-Verlag, 1971.

29. Hyman Bass, Algebraic K-theory, Benjamin, New York, 1968.

30. F. R. Gantmakher, The theory of matrices, 2nd ed., "Nauka", Moscow, 1966; English transl. of 1st ed., vols. 1, 2, Chelsea, New York, 1959. 